\title{
Quantifying uncertainties in signal position in non-resolved images: application to space object observation
}

\author{
Francois Sanson ${ }^{\mathrm{a}, *}$, Carolin Frueh ${ }^{\mathrm{b}}$ \\ ${ }^{a}$ Inria Bordeaux Sud-Ouest, 200 Rue de la Vieille Tour, 33405 Talence, France \\ ${ }^{b}$ Assistant Professor at School of Aeronautics and Astronautics, Purdue University, West \\ Lafayette IN 47906, USA
}

\begin{abstract}
Charged Coupled Devices (CCDs) and subsequently Complementary metaloxidesemiconductor (CMOS) detectors revolutionized scientific imaging. On both the CCD and CMOS detector, the incoming photons release photo electrons that are then read out to produce an image. Unfortunately the generated images are degraded by inevitable noise introduced by the stochastic nature of the electron emission process and the read out process. Moreover, in many applications, such as in astronomy or for satellite tracking, only unresolved object images are available due to optical diffraction. It therefore is of integral interest to determine the center or so-called centroid of the non-resolved signal with a sub-pixel precision. The most common method for centroid estimation is to fit Gaussians or other parametrized surfaces to the signal in order to find the center of mass of the light. Because of the detector noise, the centroid estimate is necessarily uncertain. While the uncertainty in the centroid is classically estimated by running prohibitively costly Monte Carlo simulations, in this paper, we propose analytic uncertainty estimates of the centroid position. The expressions that depend on the pixel size, the signal to noise ratio and the extension of the object signal relative to the pixel size are validated against rigorous Monte Carlo simulations with very satisfying results. Numerical tests
\end{abstract}

\footnotetext{
*Corresponding author. Tel.: +33(0)5 24574112

Email address: francois.sanson@inria.fr (Francois Sanson)
}

Preprint submitted to Advances in Space Research

April 18, 2018 
show that our analytic expression is an efficient substitute to the Monte Carlo simulation thereby reducing computational cost.

Keywords: Space Situational Awareness

\section{Introduction}

Space object observation heavily relies on Charged Coupled Devices (CCDs) and Complementary MetalOxideSemiconductor (CMOS) detectors. Both detectors are based on the same principle. In the semiconductor material, which can be front or back-illuminated, photo-electrons are released upon the impact of incoming photons. In the best performance region of the sensor, the ratio between the incoming photons and the released photo-electrons is constant while in the low and upper end of the detector threshold, the proportionality tends to vary leading to what is known as the non-linearity of the sensor. One of the main differences between CCD and CMOS is the read-out process. Whereas in a CCD, charge is shifted and one line is read out at a time, a CMOS sensor uses parallel readout of the whole image is possible. Readout times hence are significantly lower for CMOS compared to same sized CCD. The image is in both cases comprised of analog-to-digital units (ADU) that, via the camera gain, transforms the photo-electrons into digital units. For images of objects that are too far away from the sensor and or too small for the optic setup, only non-resolved images are available. Broadly speaking, non-resolved images consist of one bright dot per object, that can cover one or up to several hundred pixels while not featuring any object details. Fig 1 shows a perfectly noiseless non-resolved object image. Physically speaking, the bright dot corresponds to the first maximum of the diffraction pattern, for a round aperture, the so-called Airy disk. The main maximum contains over 80 percent of the whole light received from the object. Higher order maxima are normally not discernible and hence visible on the final image.

As the sensing process, including reception of photons, release of photo-electrons and the read-out process, is taking place on the quantum level, it is by nature 
a stochastic process. This means that the image generated by a sensor differs across repeated experiments despite having exactly the same amount of light entering the detector. Classically, the detector response has been modeled as a Poisson process with the expectation value and variance corresponding to the nominal irradiation value entering the detector [1. An example of four synthetically generated representations of the same irradiation entering the detector are plotted in Fig. 2, Besides the stochastic sensing process of the irradiation received from the object, there are two additional types of noise sources that corrupt the object images and impact object image detection and position estimation. One source is the other external light sources that enter the detector with the light emitted or reflected off the object of interest. This spurious light creates photo electrons identical to irradiation from the object of interest itself and are usually modelled as Poisson distribution. The second source comes from noise generated by the detector itself. Given that the detector is never at zero Kelvin, thermal motion leads to so-called dark noise, that is even present when the shutter is not opened. Furthermore, there is the noise generated by the read-out process itself and the truncation error, as only integer pixel values can be recorded in ADUs. Practically speaking background noises imply that the background underneath the object images does not have a zero pixel value. Further images disruption, such as hot or dead pixels or charged particles impinging on the detector, so-called cosmics, are not discussed here.

In many applications using images featuring non-resolved signals, such as detection and tracking of stellar objects or satellites, or tracking of blood cells in microscopic imaging for example in medicine or biology, object detection and position estimation is challenging $[2,3,4,5,6,7,8$. Tracking algorithms rely on precise estimation of the signal position at the subpixel level. This is usually done via determining the center, often called centroid, of the object signal. The center is then assumed to be the object position at the mid-exposure time. Because of the image noise and the stochastic nature of the detection, the centroid location is not straightforward. In practice, there are two main classes of techniques to find the centroid of a non-resolved digital object image 


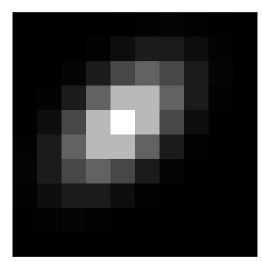

Figure 1: Noiseless signal

: one is to fit a two-dimensional function over the pixel values of the object images and its surrounding pixels while the second finds the center of light of the object image [4, 1, 9]. In the former case, for slightly distorted object images, a Gaussian function fitting is often performed using a maximum likelihood estimator [10, 11, 12, 13, 14. The latter methodology has the advantage of producing reliable results for highly distorted images, for which surface fitting often fails. Both methodologies require that the background of the images has been subtracted. Description of image processing pipelines for astrometric observations of near-Earth objects is discussed in 15, 4, 16, 17.

As with any sensing and estimation problem, the estimated centroid differs for different representations of the same irradiation reaching the detector. This is illustrated in Fig. 2 and Fig. 3. Fig. 2 shows the same object signal in four different realizations. Fig. 3 shows the histogram of pixel values, the fitted Gaussian surface and the centers that have been determined for identical irradiation reaching the detector. For the applications, it is of utmost interest to have a representation of the uncertainty associated with the centroid. The variance in the brightness of a pixel value can be computed using the inverse of the signal to noise ratio [18, 9]. On the other hand, the variance in the centroid position is not just a straightforward estimation problem.

As a result, centroid uncertainties are often determined with costly Monte Carlo methods based on simulated images. This computationally very intensive process is sought to be avoided. 
The major contribution of this paper is the derivation of an analytical approximation of centroid position variance that does not require any Monte Carlo simulations. The derived expression is thoroughly compared to alternative expensive methods such as Monte Carlo. The derived expressions are also compared to existing analytical expressions [19, 20, 21]. All the tests show that the estimation of the centroid variance is a good approximation of the Monte Carlo results even for relatively small and cropped signals. In this work, it is also shown that the previous estimates of this lower bound systematically underestimate the true variance, based upon the comparison with Monte Carlo simulations. For object images, which are spread over less than four pixels, a Bayesian formulation of the problem is introduced. Realistic bounds on the object image centroid position on the sub-pixel level are provided based on the pixel scale and the signal to noise ratio of the brightest pixel. Finally, a look-up table with reference values is provided. Preliminary work on this topic has been published by the authors in $[22$.

The paper is organized as follows: In the second section, we describe the centroid estimation process of a noisy non-resolved object image on a pixel grid using Gaussian fitting. In the third section, the improved estimation of the centroid position uncertainty is shown and analytical expressions are derived. Based on the derivations, the fourth section provides the validation of the methodology via comparison with Monte Carlo simulations and Bayesian estimation. In the fifth section, application guidelines for the variance computation in observed image frames are provided. The findings are summarized in the conclusions. In Appendix A a look-up table for fast application in observations can be found. 

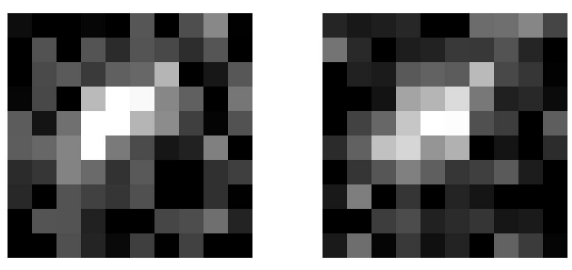

(a) Signal realization 1 (b) Signal realization 2
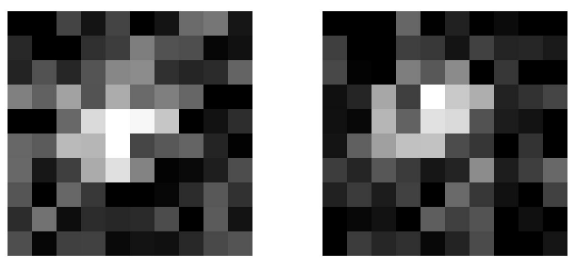

(c) Signal realization 3 (d) Signal realization 4

Figure 2: Four realizations of the same noiseless signal (cf Fig. 1] 


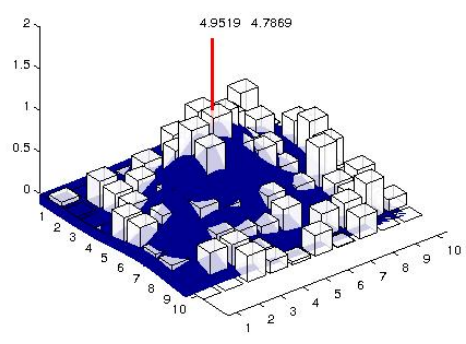

(a) Signal realization 1

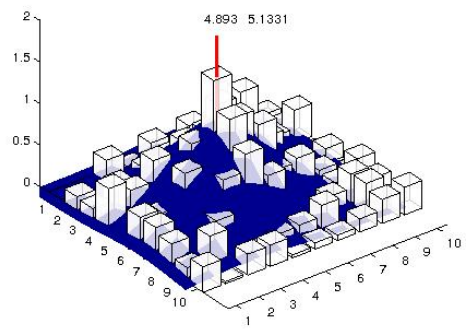

(c) Signal realization 3

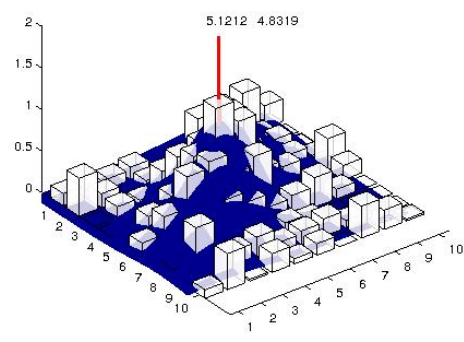

(b) Signal realization 2

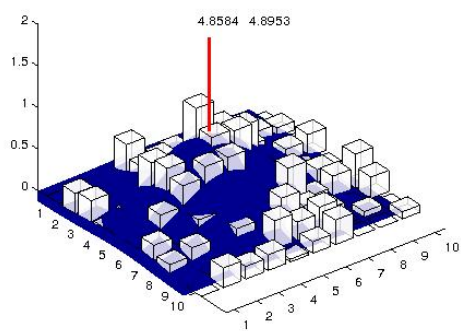

(d) Signal realization 4

Figure 3: Four realizations of the same signal (cf Fig. 1) with fitted Gaussians. The red line indicates the position of the centroid in each case 


\section{Overview of Object Image Centroiding in Noisy Images}

In this section the process of finding an object image centroid in the presence of noise is summarized. First, the expressions for the signal in the pixels containing the object image are derived. Further details on the derivation of the CCD equation and advances in the presence of ambiguous pixels can be found in [23, 22. Secondly, the process of the maximum likelihood estimation is shown. Details on centroid determination techniques via Gaussian fitting can be found in $10,11,12,13,14$.

\subsection{Signal of the Object Image Pixels}

The Gaussian or other parametrized surface is fitted to the signal after background subtraction. The total signal $S$ of all pixels containing the object image can be written as [? 22]:

$$
S=\sum_{i}^{n_{p i x}} S_{i}+R_{i}-B_{\text {est }}
$$

where $n_{p i x}$ is the number of pixels containing the object image (i.e. the refraction Airy disk). The pixels are indexed by $i . S_{i}$ is the truncated signal distribution of each pixel. The truncation process is due to the fact that only integers can be reported from the CCD during the electron to ADU conversion. Defining the gain $g$, one $\mathrm{ADU}$ corresponds to $g$ photoelectrons. $R_{i}$ is the readout noise. $B_{\text {est }}$ is the estimated background that is subtracted.

The distribution of $S_{i}$ can be understood as [23, 22]:

$$
\begin{aligned}
P\left(S_{i}=q\right) & =\sum_{k=g q}^{g(q+1)-1} \frac{\exp \left(-\lambda_{\mathrm{S}, i}\right) \lambda_{S, i}^{k-\frac{1}{2} g}}{\left(k-\frac{g}{2}\right) !} \\
& =\frac{\Gamma\left(g\left(q+\frac{1}{2}\right), \lambda_{\mathrm{S}, i}\right)}{\Gamma\left(g\left(q+\frac{1}{2}\right)\right)}-\frac{\Gamma\left(g\left(q-\frac{1}{2}\right), \lambda_{\mathrm{S}, i}\right)}{\Gamma\left(g\left(q-\frac{1}{2}\right)\right)},
\end{aligned}
$$

with $\lambda_{\mathrm{S}, i}=\lambda_{\text {ext }, i}+\lambda_{\mathrm{obj}, i}+\lambda_{\mathrm{D}, i}$ where $\lambda_{\text {ext }, i}$ is the mean intensity from the background noise entering the detector as external light sources that are not from the object of interest. An example in astronomy would be the stellar background 
sources. $\lambda_{\mathrm{D}, i}$ denotes the mean intensity of the dark noise and $\lambda_{\mathrm{obj}, i}$ is the mean intensity coming from the object itself. $\Gamma$ is the gamma function defined as $\Gamma(n, x)=\int_{x}^{\infty} e^{-t} t^{n-1} d t$ and $\Gamma(n)=(n-1)$ !. Details of the derivation can be found in 23 .

Finally, $B_{\text {est }}$ is the estimated background defined in [22, 9] as :

$$
B_{\mathrm{est}}=\frac{1}{n_{B}} \sum_{i}^{n_{\mathrm{B}}} S_{i}+R_{i}
$$

with $n_{B}$ is the number of pixels used for the background estimation.

Eq. 1 is the sum of random variables that follow different distributions. The following assumptions are made for simplification in order to approximate Eq. 1 as a sum of Gaussian random variables. Assuming that the gain is small or alternatively that the CCD resolution is extremely high (hypothesis 1) the truncated Poisson $S_{i}$ can be interpreted as a Poisson random variable with a good level of accuracy. For he actual signal on the CCD, it can be assumed that the mean signal intensity is such that $\lambda_{\mathrm{ext}, i}+\lambda_{\mathrm{obj}, i}+\lambda_{\mathrm{D}, i} \gg 1 \forall i$ (hypothesis 2). The use of the Central Limit Theorem can account for modelling both, the signal and the background as normal distributions: $S_{i} \sim \mathcal{N}\left(\lambda_{\mathrm{ext}, i}+\lambda_{\mathrm{obj}, i}+\lambda_{\mathrm{D}, i}, \sqrt{\lambda_{\mathrm{ext}, i}+\lambda_{\mathrm{obj}, i}+\lambda_{\mathrm{D}, i}}\right)$ and $B_{\text {est }} \sim \mathcal{N}\left(\lambda_{\text {ext }, i}+\lambda_{\mathrm{D}, i}, \sqrt{\frac{\lambda_{\text {ext }, i}+\lambda_{\mathrm{D}, i}}{n_{b}}}\right)$. In the latter, the number of pixels used in the background determination $n_{B}$ is assumed to be large (hypothesis 3), which is advantageous for precise background determination, as shown in Eq3. Note that in order to preserve the homogeneity of $S$, we denote $\operatorname{Var}(S)=\lambda_{\mathrm{S}, i} \times 1_{s}$, where $1_{s}$ only carries the unit of energy. $R_{i}$ can be taken to be Gaussian as well [9, 18, and is assumed to be constant over the signal pixels (hypothesis 4). Whereas the latter is a good approximation for a CCD, the assumption might be violated for object images spread out over a large number of pixel on a CMOS detector. Finally under the assumption previously stated, the effective signal in the $i^{t h}$ pixel $S_{i}$ as in Eq. 1 is the sum of three independent Gaussian random variables:

$$
S_{i}=\lambda_{\mathrm{S}, i}-\lambda_{\mathrm{ext}, i}+\lambda_{\mathrm{D}, i}+\mathcal{N}\left(0,1_{s} \times \sqrt{\lambda_{\mathrm{S}, i}+\frac{\lambda_{\mathrm{ext}, i}+\lambda_{\mathrm{D}, i}}{n_{b}}+\sigma_{R}^{2}}\right),
$$


where $\sigma_{R}^{2}$ is the variance of the readout noise at the $i$ th pixel. The Gaussian distribution signal distribution assumptions will become crucial when determining explicit expressions of the Fisher information.

\subsection{Maximum Likelihood Centroid Estimation}

The background subtracted object image signal pattern at the sensor (see Eq. 33 is fitted with a Gaussian curve. An example of Gaussian fitting applied to an actual signal is given in Fig. 3. For details see 24. The actual position of the source can be retrieved under the assumption that it corresponds to the center of the fitted Gaussian curve on the pixel grid. This is in general not the center of any given pixel. More precisely,it is assumed that the fitted subspace has the following form

$$
G=A e^{\left(-\frac{1}{2}\left(c_{1}\left(x_{i}-x_{0}\right)^{2}+2 c_{3}\left(x_{i}-x_{0}\right)\left(y_{i}-y_{0}\right)+c_{2}\left(y_{i}-y_{0}\right)^{2}\right)\right)},
$$

noting $\theta=\left(A, x_{0}, y_{0}, c_{1}, c_{2}, c_{3}\right)$ where $A$ is the amplitude, $x_{0}$ and $y_{0}$ the center of the fitted Gaussian on the pixel grid and $c_{1}, c_{2}$ and $c_{3}$ the coefficients of the inverse covariance matrix. The position of the object image on the pixel grid then is denoted by $x_{0}, y_{0}$. A note on the units: $x_{0}, y_{0}, x, y$ have the same unit as pixels, but allow for sub-pixel values for the center of the Gaussian. The angles can be given in arcseconds. $c_{1}, c_{2}, c_{3}$ are expressed in $\operatorname{arcsec}^{-2}$ and $A$ is a number of electrons per $\operatorname{arcsec}^{2}$. The pixel scale (arcseconds per pixel) is assumed to be known.

The maximum of likelihood (ML) estimator has been widely used in Gaussian fitting of signals [19, 10, 25] for its good properties: it is an unbiased, consistent and asymptotically efficient estimator under mild assumptions [26]. Defining the likelihood of the set of parameters $\theta$ for a signal as:

$$
L=P(S=G \mid \theta),
$$

then the maximum of likelihood is defined as:

$$
\hat{\theta}_{M L E}=\operatorname{argmax}_{\theta}(P(S=G \mid \theta)) .
$$


If the noise is Gaussian independent for two different pixels, the likelihood becomes:

$$
L=\prod_{i}^{n_{\mathrm{pix}}} \exp \left(-\frac{1}{2}\left(\frac{\left(S_{i}-G_{i}\right)^{2}}{\sigma_{i}^{2}}\right)\right) .
$$

$G_{i}$ is the Gaussian surface, Eq. 5 and $S_{i}$ signal at the $i$ th pixel, defined in Eq1. $\sigma_{i}$ is the standard deviation of the noise distribution at pixel $i$. If the noise amplitude is assumed to be constant over the signal $\sigma_{i}$ is constant and denoted $\sigma$ in the rest of this article. Then the log likelihood also called the score is:

$$
l=-\sum_{i}^{n_{\text {pix }}} \frac{\left(S_{i}-G_{i}(\theta)\right)^{2}}{2 \sigma^{2}} .
$$

\section{Uncertainty Quantification of the Centroid}

In this section we derive analytical uncertainty estimates of the signal center estimation. The Gaussian fitting can only be applied to a specific realization of the stochastic object image generation process according to the distributions of the terms in Eq3. Under the assumptions discussed in the previous section, a sum of Gaussian random variables, Eq4. As discussed in the introduction and illustrated Fig 2 there, different realizations lead to different centroids. Fig 3 shows the 3D representation of noise affected images with the fitted Gaussian surface.

We present two common approaches to quantify the uncertainty in the estimation of the parameters : a maximum of likelihood estimation as it is carried in [19, 27] or a Bayesian approach [28. Both methods tackle a problem from a different angle so the uncertainty in the estimation are in general different. The maximum of likelihood estimation focuses on estimating the deterministic parameters $\theta_{\text {true }}$ and then estimates the uncertainty in the estimator $\hat{\boldsymbol{\theta}}$. On the contrary, with the Bayesian approach, one considers the distribution of $\Theta$ for a given set of measurements. Therefore, even if uniform priors are used for the Bayesian estimation both cases are not exactly equivalent and yield different results for small signal. 
When fitting a Gaussian surface to the signal, one needs to estimate $\theta$, which holds the six Gaussian surface parameters, namely, $A, c_{1}, c_{2}, c_{3}$ along with the centroid $x_{0}, y_{0}$. However, often in imaging, only the centroid, comprising of two parameters are of major interest. Hence, the first four parameters may be treated as nuisance parameters. The nuisance parameter estimation not only influences the nominal values of the remaining quantities of interest, but also affects the variance in the parameters of interest. Uncertainty in their estimation leads to increased uncertainty in the parameters of interest. As stated in [29] and 30 the variance with nuisance parameters is always larger than without nuisance parameters.

\subsection{Rao-Cramer lower bound}

Under regularity assumptions on the likelihood function, for any unbiased estimator, there exists a variance lower bound [26]. In our case, the Maximum of Likelihood (ML) estimator asymptotically reaches this lower bound [26, 30] and therefore this lower bound can be used as an approximation of the centroid position variance. We first introduce the Fisher information as the expectation value of the Hessian of the score function defined in Eq. 9 .

$$
F(\theta)=\mathbb{E}\left[\frac{\partial^{2} l}{\partial^{2} \theta_{i} \theta_{j}}\right],
$$

where $\mathbb{E}$ denotes expected value with respect to the likelihood. As explained in [26], the Fisher information corresponds to the average amount of information available in the sample. The Fisher information as derived in [31] is given in Appendix B. The Rao Cramer lower bound (RCLB) variance is related through the following inequality to the Fisher information:

$$
\operatorname{Var}_{s_{1} . . s_{n}}(\hat{\theta}) \geq F(\theta)^{-1} .
$$

Which means that the variance of the Rao Camer lower bound is always larger or equal to the inverse of the Fisher information gain. If the number of pixels used in the ML estimation is sufficiently large, the ML estimator converges toward a Gaussian distribution ( [27, 30]):

$$
\hat{\theta} \sim \mathcal{N}\left(\theta_{\text {true }}, F(\theta)^{-1}\right) .
$$


In that case, the lower bound will be reached, the inequality then becomes an equality and a good analytical estimation of the RCLB variance becomes available.

In many cases, simple analytical expressions of the Fisher information are not available, but in our case, previous works [20, 19] have managed to derive such expression under the following assumptions:

1. Flat noise: $\sigma$ is constant over all pixels of the signal.

2. The profile is well sampled: the pixel size is constant and small compared to the object image size. In other words we have $\delta_{x}, \delta_{y} \ll \frac{1}{c_{1}^{0.5}}, \frac{1}{c_{2}^{0.5}}$ where $\delta_{x}$ and $\delta_{y}$ are the CCD pixel dimensions.

3. The entire profile of the object image is sampled

4. Centroid of the signal is at the center of a pixel. In other words: $\xi_{n}=\delta_{x} n$ and $\eta_{m}=\delta_{y} m$

5. The signal distribution is Gaussian within each pixel

The profile of the object image when approximated with a Gaussian is theoretically infinitely wide spread over the whole pixel grid. The exact expression for the Fisher information as derived in [31] and can be found in Appendix B. The Fisher information terms can be decomposed into sums of the form:

$$
\sum_{m, n=0}^{n_{\text {pix }}} R_{m, n} \xi_{n}^{i} \eta_{m}^{j} \text { with } j, i \leq 4
$$

where $R_{n, m}=\frac{\delta_{x}^{2} \delta_{y}^{2} E_{n, m}^{2}}{2 \sigma^{2}}$ and

$E_{m}=\exp \left(-\frac{1}{2}\left(c_{1}\left(x_{n}-x_{0}\right)^{2}+2 c_{3}\left(x_{n}-x_{0}\right)\left(y_{m}-y_{0}\right)+c_{2}\left(y_{m}-y_{0}\right)^{2}\right)\right), \xi_{n}=$ $x_{n}-x_{0}$ and $\eta_{m}=y_{m}-y_{0}$.

The task now is to compute the sums. Their derivation can be involved so most of the derivation is left in Appendix D and Appendix C while this paragraph focuses on the assumptions made to carry out the calculations. Under the hypotheses one through four above, [19] the sums can be approximated 
with their corresponding integrals; this approach was already used in [25]. This approximation is justified by classical results on Riemann integrals and accurate up to the second order in $\delta_{x}, \delta_{y}$, the pixel size in $x$ and $y$ directions. However, it is found that the assumptions one through four are not well justified : assumption one is never fully satisfied, except for small object image traces relative to the background variation. Hypothesis two neglects the cutoff effects for extremely under-sampled object images. The third assumption used in [19] is nearly impossible to comply with in practice. Firstly, when there is more than one object image in the whole observation frame, the object image is cropped over a finite number of pixels. Furthermore, the background necessarily changes across the domain and clashes with hypothesis one. In practice, a sub-frame technique with limited size sub-frames is used and hence assumption three is violated. Assumption 4 is in general violated. As the irradiation enters the detector unrestricted, the centroid is only in rare cases at the center of a pixel or symmetric to the pixel grid but can be anywhere within the pixel grid. This is also confirmed by real observations [17]. In general, we have $\eta_{m}=u_{y}+\delta_{y} m$ and $\xi_{n}=u_{x}+\delta_{x} n$, where $u_{x}$ and $u_{y} \in\left[0, \frac{\delta_{x}}{2}\right) \times\left[0, \frac{\delta_{x}}{2}\right)$.

In order to relax the assumptions to better fit the conditions of actual observations, a truncated signal is assumed not symmetric with the pixel grid, avoiding assumptions three and four, while keeping assumptions one, two and five.

Denoting $a$ and $b$ as the cropping boundaries (ie. the portion of the considered signal lies in the rectangle $[-a ; a] \times[-b ; b])$, it is further assumed that $a \sqrt{\frac{D}{c_{2}}} \gg 1$ and $b \sqrt{\frac{D}{c_{1}}} \gg 1$. Note that $D=c_{1} c_{2}-c_{3}^{2}$. Due to this unsymmetrical distribution of the pixels around the center of the object and the cropping around the centroid of the object image, the substitution of sums with their integrals is only accurate up to the first order in $\delta_{x}, \delta_{y}$ for some terms of the Fisher information. In order to get improved analytical expressions for Eq. 15, $u_{x}$ and $u_{y}$ are unknown a priori. They are modeled with a uniform distribution $\mathcal{U}\left[0, \frac{\delta_{x}}{2}\right]\left(\operatorname{resp} . \mathcal{U}\left[0, \frac{\delta_{y}}{2}\right]\right)$ 25] and the sums are approximated with its corresponding integral. A change of 
variable yields the following approximation for $\sum_{n} \sum_{m} R_{n, m} \xi_{n}^{i} \eta_{m}^{j}$ with a second order accuracy in the pixel size $\delta_{x, y}$ :

$$
\sum_{n} \sum_{m} R_{n, m} \xi_{n}^{i} \eta_{m}^{j} \approx J_{x^{i} y^{j}}=\int_{-a+u_{x}}^{a+u_{x}} \int_{-b+u_{y}}^{b+u_{y}} x^{i} y^{j} e^{\left(-\left(c_{1} x^{2}+2 c_{3} x y+c_{2} y^{2}\right)\right)} d x d y
$$

This approximation is key to derive an analytical expression for the RCLB. The calculations in the rest of this section aim at deriving analytical expressions for $J_{x^{i} y^{j}}$. Using Eq. 14, the $J_{x^{i} y^{j}}$ are then injected in the analytical expression for the Fisher Information Eq. B.1 from which the RCLB can be derived.

Note: The Fisher information computed in [19] only uses the constant terms in $\delta\left(\right.$ order $\left.0^{\text {th }}\right)$. Unfortunately, this approximation leads to a covariance matrix where the position parameters $\left(x_{0}, y_{0}\right)$ and the nuisance parameters appear to be uncorrelated, which we know is untrue. In the appendix it is shown that, in general

$$
\sum_{m=0}^{n_{\text {pix }}} R_{m} \xi_{n}^{i} \eta_{m}^{j}=\sum_{n} \sum_{m} R_{n, m} \xi_{n}^{i} \eta_{m}^{j}=0+\epsilon\left(u_{x}, u_{y}\right), \text { if } i+j \text { is odd, }
$$

with $\epsilon \propto \delta_{x, y}$. The sum in Eq. 15 is equal to zero, when the signal/object image is uncropped or $u_{x}$ and $u_{y}$ are non zero. Because we do assume a cropped signal, all the terms, where $i+j$ are even and when $i+j$ are odd they are non-zero.

Derivation of analytic expression of $J_{x^{i} y^{j}}$ (Eq. 14), when $i+j$ is odd

We define

$$
f_{i, j}(x, y)=x^{i} y^{j} e^{\left(-\left(c_{1} x^{2}+2 c_{3} x y+c_{2} y^{2}\right)\right)}
$$

using the symmetries of $f_{i, j}$, we have $\int_{\left[0, a-u_{x}\right] \times\left[0, b-u_{y}\right]} f_{i, j}(x, y) d x d y=$ $-\int_{\left[0,-a+u_{x}\right] \times\left[0,-b+u_{y}\right]} f_{i, j}(x, y) d x d y$ and $\int_{\left[0,-a+u_{x}\right] \times\left[0, b-u_{y}\right]} f_{i, j}(x, y) d x d y=-\int_{\left[0, a-u_{x}\right] \times\left[0,-b+u_{y}\right]} f_{i, j}(x, y) d x d y$. The integral in Eq14 can hence be decomposed and simplified into:

$$
J_{x^{i} y^{j}}=\int_{a-u_{x}}^{a+u_{x}} \int_{-b}^{b} f_{i, j}(x, y) d x d y+\int_{-a}^{a} \int_{b-u_{y}}^{b+u_{y}} f_{i, j}(x, y) d x d y
$$


Note: The square of size $\left[a, a+u_{x}\right] \times\left[b, b+u_{y}\right]$ is included and $\left[a-u_{x}, a\right] \times\left[b-u_{y}, b\right]$ is counted twice but they are small (order two in the pixel size). Assuming $u_{x}$ and $u_{y}$ are small, Taylor series expansions around $a$ and $b$ leads to the following expression:

$$
\begin{aligned}
J_{x^{i} y^{j}} & =2 u_{x} a^{i} \exp \left(-\frac{a^{2} D}{c_{2}}\right) \int_{-b}^{b} y^{j} \exp \left(-\frac{y^{2} D}{c_{1}}\right) d y \\
& +2 u_{y} b^{j} \exp \left(-\frac{b^{2} D}{c_{1}}\right) \int_{-a}^{a} x^{i} \exp \left(-\frac{x^{2} D}{c_{2}}\right) d x .
\end{aligned}
$$

Eq. 18 shows that $J_{x^{i} y^{j}}$ scales like $u_{x} a^{i} \exp \left(-\frac{a^{2} D}{c_{2}}\right)$ that should be small according to our hypotheses. For completeness, full calculations of the integrals are provided in Appendix C, however their exact expressions are not necessary for the remainder of this study as they will constitute negligible components of the Fisher information.

Computation of the $J_{x^{i} y^{j}}$ (Eq. 14), when $i+j$ is even

We now focus on the even integrals ie. the integrals for which $i+j$ is even by computing:

$$
J_{x^{i} y^{j}}=\int_{-a+u_{x}}^{a+u_{x}} \int_{-b+u_{y}}^{b+u_{y}} x^{i} y^{j} e^{\left(-\left(c_{1} x^{2}+2 c_{3} x y+c_{2} y^{2}\right)\right)} d x d y .
$$

Unfortunately, no simpler analytical expressions are available for truncated Gaussian integrals contrary to non truncated Gaussian integrals. Also,

$$
J_{x^{i} y^{j}}=N_{x^{i} y^{j}}-M_{x^{i} y^{j}}
$$

where

$$
N_{x^{i} y^{j}}=\int_{-\infty}^{\infty} \int_{-\infty}^{\infty} x^{i} y^{j} e^{\left(-\left(c_{1} x^{2}+2 c_{3} x y+c_{2} y^{2}\right)\right)} d x d y
$$

and

$$
M_{x^{i} y^{j}}=\iint_{T} x^{i} y^{j} \exp \left(-\left(c_{1} x^{2}+2 c_{3} x y+c_{2} y^{2}\right)\right) d x d y,
$$

with $T=\left(-\infty ;-a+u_{x}\right] \cup\left[a+u_{x} ;+\infty\right) \times\left(-\infty ;-b+u_{y}\right] \cup\left[b+u_{y} ;+\infty\right) . N_{x^{i} y^{j}}$ is the integral over the entire domain for which analytical expressions are available 
in [31] and in Appendix E. Therefore, it is enough to compute the surplus $M_{x^{i} y^{j}}$

$$
M_{x^{i} y^{j}}=\int_{-a}^{a} \int_{[-\infty ;-b] \cup[b ;+\infty]} x^{i} y^{j} \exp \left(-\left(c_{1} x^{2}+2 c_{3} x y+c_{2} y^{2}\right)\right) d x d y,
$$

While no analytical expressions are available for $M_{x^{i} y^{j}}$, we propose the following upperbound. The surplus is centered with respect to the origin leading to a second order error in the calculation. Using the symmetry $f_{i, j}(x, y)=f_{i, j}(-x,-y)$, and adding $\int_{a}^{\infty} \int_{b}^{\infty} f_{i, j}(x, y) d x d y+\int_{a}^{\infty} \int_{-\infty}^{-b} f_{i, j}(x, y) d x d y+\int_{-\infty}^{-a} \int_{b}^{\infty} f_{i, j}(x, y) d x d y+$ $\int_{-\infty}^{-a} \int_{-\infty}^{-b} f_{i, j}(x, y) d x d y>0$ (since $i+j$ is even), the surplus is bounded by :

$$
M_{x^{i} y^{j}} \leq \tilde{M}_{x^{i} y^{j}}
$$

with

$$
\begin{aligned}
\tilde{M}_{x^{i} y^{j}} & =2 \int_{-\infty}^{-a} \int_{R} x^{i} y^{j} e^{\left(-\left(c_{1} x^{2}+2 c_{3} x y+c_{2} y^{2}\right)\right)} d x d y \\
& +2 \int_{R} \int_{-\infty}^{-b} x^{i} y^{j} e^{\left(-\left(c_{1} x^{2}+2 c_{3} x y+c_{2} y^{2}\right)\right)} d x d y .
\end{aligned}
$$

If $a$ and $b$ are large relative to $\sqrt{\frac{c_{2}}{D}}$ and $\sqrt{\frac{c_{1}}{D}}$, Eq. 24 gets close to equality so $\tilde{M}_{x^{i} y^{j}}$ can be used instead of $M_{x^{i} y^{j}}$. Since we overestimate the surplus $M_{x^{i} y^{j}}$, the Fisher information is underestimated. As a result the variance is overestimated. The advantage of using $\tilde{M}_{x^{i} y^{j}}$ over $M_{x^{i} y^{j}}$ is that the integrals have exact analytical expressions in $\tilde{M}_{x^{i} y^{j}}$. For instance $\tilde{M}_{x^{2}}$ can be computed directly, as outlined in Appendix C and Appendix D.

Once all the $J_{x^{i} y^{j}}$ are computed, the Fisher information matrix is computed using Eq. 14 and Appendix B.

\subsection{Marginalization over the nuisance parameters}

Since only the position variances of $x_{0}, y_{0}$ are of interest, it is beneficial to compute the marginalized distribution over the nuisance parameters $A, c_{1}, c_{2}$ and $c_{3}$, that are respectively the amplitude and shape parameters of the object image. Assuming the ML estimator is normally distributed with covariance matrix $K$, the marginalized Fisher information for $x_{0}$ and $y_{0}$ is [29]

$$
F_{x_{0}, y_{0}}^{\text {marg }}=F_{x_{0}, y_{0}}-F_{\left[x_{0}, y_{0}\right],\left[A, c_{1}, c_{2}, c_{3}\right]} F_{A, c_{1}, c_{2}, c_{3}}^{-1} F_{\left[x_{0}, y_{0}\right],\left[A, c_{1}, c_{2}, c_{3}\right]}^{T} .
$$


Eq. 26 shows that the presence of nuisance parameters leads to a loss of information as $F_{x_{0}, y_{0}}^{\operatorname{marg}} \leq F_{x_{0}, y_{0}}$. Components of $F_{\left[x_{0}, y_{0}\right],\left[A, c_{1}, c_{2}, c_{3}\right]}$ are sums of the form $\sum x_{n}^{i} y_{m}^{j} \exp \left(-c_{1} x_{n}^{2}-c_{2} y_{m}^{2}-2 c_{3} x_{n} y_{m}\right)$, for which $i+j$ is odd. It has been shown (see Eq 18 that the later sums are of the same order as $u_{x} \exp \left(-\frac{a^{2} D}{c_{2}}\right)$. It follows therefore that every component of $F_{\left[x_{0}, y_{0}\right],\left[A, c_{1}, c_{2}, c_{3}\right]}$ $\times F_{A, c_{1}, c_{2}, c_{3}}^{-1} \times F_{\left[x_{0}, y_{0}\right],\left[A, c_{1}, c_{2}, c_{3}\right]}^{T}$ is of the same magnitude as $u_{x}^{2} \exp \left(-2 \frac{a^{2} D}{c_{2}}\right)$. Therefore, the contribution of the nuisance parameters in the Fisher information is extremely small and will be neglected.

Taking the inverse of $F_{x_{0}, y_{0}} \simeq F_{x_{0}, y_{0}}^{\operatorname{marg}}$, the Rao Cramer lower bound has the following form:

$$
\begin{aligned}
K_{x_{0}, x_{0}} & =\frac{2 \sigma^{2}\left(c_{1} c_{2} \sqrt{\pi}-2 D \sqrt{\frac{D}{c_{1}}} b d_{b}\right) c_{2}}{Q}, \\
K_{x_{0}, y_{0}} & =-\frac{2 c_{3} c_{1} c_{2} \sigma^{2}}{Q} \\
K_{y_{0}, y_{0}} & =\frac{2 \sigma^{2}\left(c_{1} c_{2} \sqrt{\pi}-2 D \sqrt{\frac{D}{c_{2}}} a d_{a}\right) c_{1}}{Q},
\end{aligned}
$$

With $\sigma^{2}$ being the signal variance and

$$
Q=\sqrt{\pi} A^{2} \sqrt{D}\left(4 \frac{D^{2}}{\sqrt{c_{2} c_{1}}} a d_{a} b d_{b}-2 c_{1} c_{2}\left(\sqrt{\frac{D}{c_{2}}} a d_{a}+\sqrt{\frac{D}{c_{1}}} b d_{b}\right)+c_{1} \pi c_{2}\right) \delta_{x} \delta_{y}
$$

$d_{a}=\exp \left(-\frac{D a^{2}}{c_{2}} D\right)$ and $d_{b}=\exp \left(-\frac{D b^{2}}{c_{1}} D\right)$. In order to further simplify Eq. [31. it is convenient to introduce the signal to noise ratio of the brightest pixel as defined in [23]. Under the assumptions used to derive $\mathrm{Eg} 31$, it can be written as:

$$
\frac{S_{b}}{N}=\frac{A \exp \left(-\frac{1}{2}\left(c_{1} u_{x}^{2}+2 c_{3} u_{x} u_{y}+c_{2} u_{y}^{2}\right)\right) \delta_{x} \delta_{y}}{\sigma},
$$

where $u_{x}$ and $u_{y}$ were previously defined as the minimum distance between the center of the Gaussian surface and the center of a pixel for a given signal. Eq. 30 does not take into account the pixel integration as in the derivation of Eq. 27. It is possible to average $u_{x}$ and $u_{y}$ as in the derivation of the Rao Cramer Lower 
Bound, however, to simplify $u_{x}$ and $u_{y}$ are set to zero so Eq. 29 becomes:

$$
\frac{S_{b}}{N}=\frac{A \delta_{x} \delta_{y}}{\sigma}
$$

and keeping only first order terms Eq. 27 becomes :

$$
\begin{aligned}
K_{x_{0}, y_{0}}= & \frac{\delta_{x} \delta_{y}}{\left(S_{b} / N\right)^{2} \sqrt{D} \pi^{3 / 2}} \times \\
& {\left[\begin{array}{cc}
2 c_{2}\left(\sqrt{\pi}+2 \sqrt{\frac{D}{c_{2}}} a d_{a}+2 \sqrt{\frac{D}{c_{1}}} \rho^{2} b d_{b}\right) & -2 c_{3}\left(\sqrt{\pi}+2 \sqrt{\frac{D}{c_{2}}} a d_{a}+2 \sqrt{\frac{D}{c_{1}}} b d_{b}\right) \\
-2 c_{3}\left(\sqrt{\pi}+2 \sqrt{\frac{D}{c_{2}}} a d_{a}+2 \sqrt{\frac{D}{c_{1}}} b d_{b}\right) & 2 c_{1}\left(\sqrt{\pi}+2 \sqrt{\frac{D}{c_{1}}} b d_{b}+2 \sqrt{\frac{D}{c_{2}}} \rho^{2} a d_{a}\right)
\end{array}\right], }
\end{aligned}
$$

where $\rho=\frac{c_{3}}{\sqrt{c_{1} c_{2}}}$ is a correlation factor between the $\mathrm{x}$ and $\mathrm{y}$ axis. It defines the orientation of the (elongated) Gaussian with respect to the pixel grid. Coefficients $c_{1}$ and $c_{2}$ are parameters of the fitted Gaussian curve that quantify the size of the signal in $x$ and $y$ direction while $c_{3}$ accounts for the orientation of the signal with respect to the axes. Variable $A$ is the intensity of the brightest pixel and $D$ is defined as $D=c_{1} c_{2}-c_{3}^{2}$. Fig 4 illustrates the elongated object image with a rotation relative to the axis and a round object image. $d_{a}=\exp \left(-\frac{D a^{2}}{c_{2}} D\right)$ and $d_{b}=\exp \left(-\frac{D b^{2}}{c_{1}} D\right)$ accounts for the truncation of the signal. If the complete object image is sampled with infinite frame bounds, $d_{b}$ and $d_{a}$ go to zero and the variance simplifies to the results developed in [19]. For actual observations, finite cropping has to be applied.

If the signal is rotation invariant as in Fig $4 \mathrm{~b}$ then $c 1=c 2=c$ and $c 3=0$ and Eq. 31 becomes:

$$
K_{x_{0}, y_{0}}=\frac{\sigma^{2}}{A^{2} \pi^{3 / 2}}\left[\begin{array}{cc}
2\left(\sqrt{\pi}+2 \sqrt{c} a d_{a}\right) & 0 \\
0 & 2\left(\sqrt{\pi}+2 \sqrt{c} b d_{b}\right)
\end{array}\right] .
$$

Introducing the signal to noise ratio as defined in Eq. 30, assuming square pixels and symmetrical cropping (ie. $a=b$ ) Eq. 32 becomes :

$$
K_{x_{0}, y_{0}}=\frac{\delta^{2}}{\pi^{3 / 2}\left(S_{b} / N\right)^{2}}\left[\begin{array}{cc}
2\left(\sqrt{\pi}+2 \sqrt{c} a d_{a}\right) & 0 \\
0 & 2\left(\sqrt{\pi}+2 \sqrt{c} a d_{a}\right)
\end{array}\right],
$$

which is a completely symmetrical expression in the $x$ and $y$ direction. 


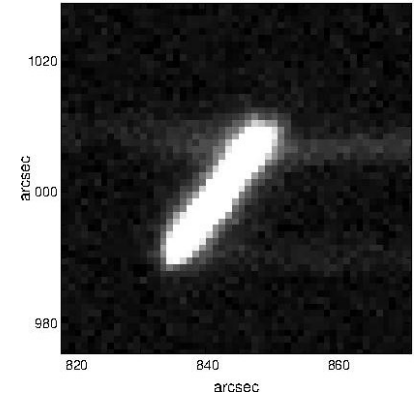

(a) Signal in the general case where $c_{1} \neq c_{2}$ and $c_{3} \neq 0$ (source ZIMLAT,AIUB)

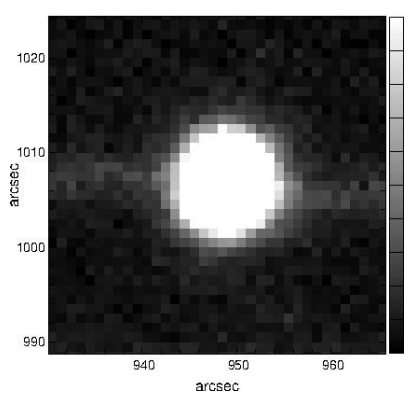

(b) Example of rotation invariant signal where $c_{1}=c_{2}$ and $c_{3}=0$ (source ZIMLAT,AIUB)

Figure 4: Signals received from an object.

\subsection{Bayesian approach}

As a comparison, a Bayesian approach is used to estimate the object's astrometric position. In this section, the joint distribution of the parameter $\theta$ given a set of pixel value is derived using exactly the same assumptions made for deriving the RCLB. Using Bayes rule we have:

$$
P\left(\theta \mid g_{1} \ldots g_{n_{\text {pix }}}\right) \propto P\left(\theta \mid g_{1} \ldots g_{n_{\text {pix }}}\right) P(\theta)
$$

with $P\left(\theta \mid g_{1} \ldots g_{n_{\text {pix }}}\right)$ referring to the posterior distribution and $P(\theta)$ the prior distribution. The ML estimator does not include the notion of prior and therefore uniform priors are taken in the Bayesian analysis. The normalized distribution $\theta \mid g_{1} \ldots g_{n_{\text {pix }}}$ was computed using Metropolis Hasting algorithm based on Monte Carlo Markov Chain (MCMC). Our implementation follows the algorithm presented in [32. The Bayesian approach computes the variance for a specific realization. The MCMC approach does not solve for the posterior directly, but only up to a scaling factor. The variance computed for example with a Monte Carlo method is obtained by averaging over multiple realizations of the same object signal. 


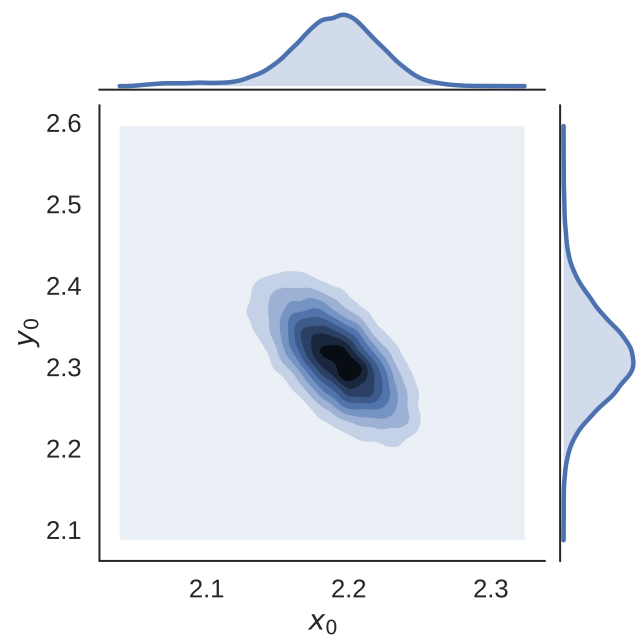

Figure 5: Joint distribution of $x_{0}$ and $y_{0}$ for a 4 pixel signal

\section{Method Comparison and Evaluation}

\subsection{Posterior Distribution}

Using the Bayesian estimation allows to determine the distribution of the object image's astrometric position even for extremely small object images (under 5 pixels above the background level). Fig 5 shows the distribution of $y_{0}$ for an object image of pixel size 4 .

Since the distribution shown in Fig. 5 is symmetrical with fast decay of its tails it seems sufficient to know the first two moments of the distribution to accurately represent the probability density function of the astrometric position estimate from the object image. Further computations for larger signals show that the astrometric distribution becomes more Gaussian as the number of integrated pixels increases, in agreement with MLE theory [26. It is hence sufficient to merely consider the variance to adequately quantify the uncertainty in the centroid location. 


\subsection{Validation}

The different methods for evaluating the variance in the object image centroid on the pixel frame are compared. The first method is the simplified method developed in [19] using the RCLB under the limiting assumptions of a centroid in the center pixel and that an infinite amount of pixels is available. The second and third methods are the improved method to compute the RCLB introduced in this paper. One is the direct numerical evaluation of the RCLB as the inverse of the Fisher information matrix given in Appendix B and denoted as exact lower bound in the figures while the other one is our analytic approximate expression, derived in Eq 31 and denoted as approximate lower bound in the figures. Finally, the Bayesian estimation is fully numerical using the Metropolis Hasting algorithm [33] implementation based on MCMC [32, 34]. The MCMC method is by far more computationally demanding than previous methods since the posterior distribution is constructed using 100000 samples. In constrast, the numerical computation of the exact RCLB is straightforward as it merely requires the numerical inversion of a 6 by 6 matrix. The expression from [19] and the RCLB that has been derived in this paper are fully analytical expressions and are hence the fastest to compute.

As ground truth, a Monte Carlo simulation is used with 1000000 samples. The results for two different noise levels, $\mathrm{SNR}=30$ and $\mathrm{SNR}=5$ (relative to the brightest pixel) are shown in Figs. 6 and 7, as a function of the full width at the half maximum (FWHM) of the object image of the true object image. The variance is given in pixels ${ }^{2}$. For the case $\mathrm{SNR}=5$, the $\mathrm{MC}$ verification is not available as the Gaussian fitted technique developed in [19] is not robust for small noisy signals. The Bayesian approach gives an alternative estimation of the variance.

In general, the Bayesian approach and the $\mathrm{MC}$ verification differ for small signals as it can be seen in Fig. 6. This is due to the theoretical difference between the Bayesian framework and the MC verification. For Fig. 7 however, the Bayesian variance is averaged over all possible observations. In both cases, the object image has been cropped at two standard deviations in $x$ direction and 
2.5 standard deviations in the $y$ direction in order to simulate unsymmetrical cropping. The noise is assumed to be constant over this cropped sub-frame. For example, if the standard deviation of the fitted Gaussian is 5 pixels in the $x$ and $y$ directions, in our test case the cropped image will be centered around the signal, 40 pixels large in the $x$ direction and 50 pixels wide in $y$ direction. It can be seen that the approximation of the RCLB according to [19] constantly underestimates the variance even for very large signals, and is hence overconfident. On the other hand, our improved method captures the cropping effect well and follows the exact lower bound well. Although theory states that the Rao Cramer Lower Bound underestimates the variance of the ML estimator, Fig. 6 and 7 show that in practice the RCLB is reached even for object images with very small FWHM and low signal to noise ratios. Consequently, even for small signals (FWHM e.g 10 pixels) the approximate RCLB defined in Eq. 31 is a good estimation of the variance. This remark is all the more true that the SNR is high. For $\mathrm{SNR}=5$, it appears that even the exact RCLB underestimates the estimator variance with respect to the Bayesian approach. Using the exact expressions for the RCLB (obtained by taking the inverse of the matrix in EqB.1) only shows significant improvements for object images with FWHM lower than two pixels. Unsurprisingly, for those cases the variance sharply increases, as more and more information is lost into one pixel.

Using the results derived in the previous section, one can instantaneously estimate the uncertainty in location of a space object without simulating object image frames or actually doing Gaussian fits. In Appendix A a lookup table gives the variance of the centroid position given the signal-to-noise ratio, the number of pixels above half the brightest pixel intensity (FWHM) and the ratio of the length of the signal in the longest direction over the length in the shortest direction counting pixels above half the brightest pixel intensity.

Being able to predict the uncertainty in astrometric position also helps to gain insight on how pixel resolution and size of the signal affects the uncertainty in the object astrometric position. Fig 9 shows how the pixel resolution influences the uncertainty in signal location for four different signals shown in Fig. 8 . The 


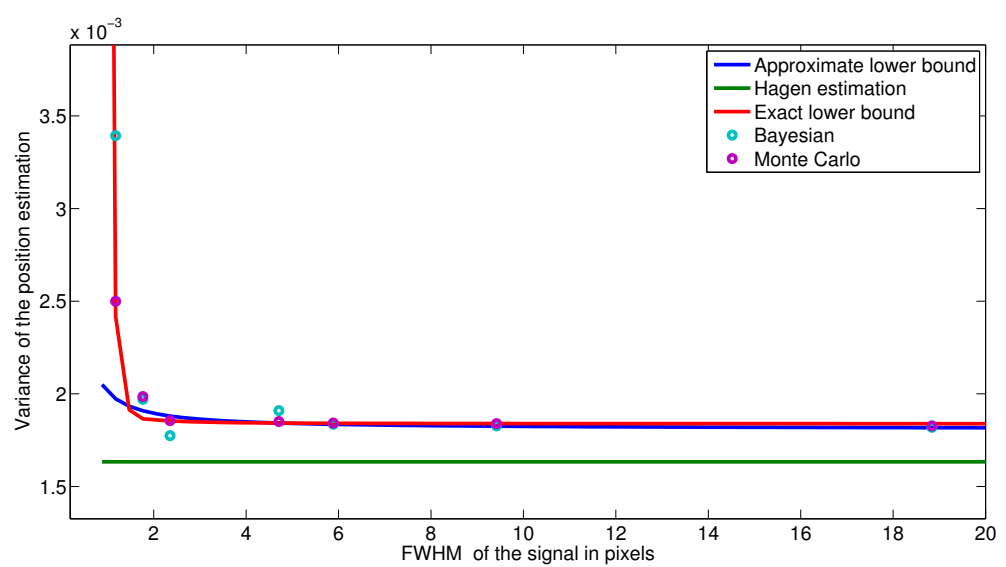

Figure 6: Convergence of the ML position estimation variance as a function of the FWHM for generated signal with $\mathrm{SNR}=30$

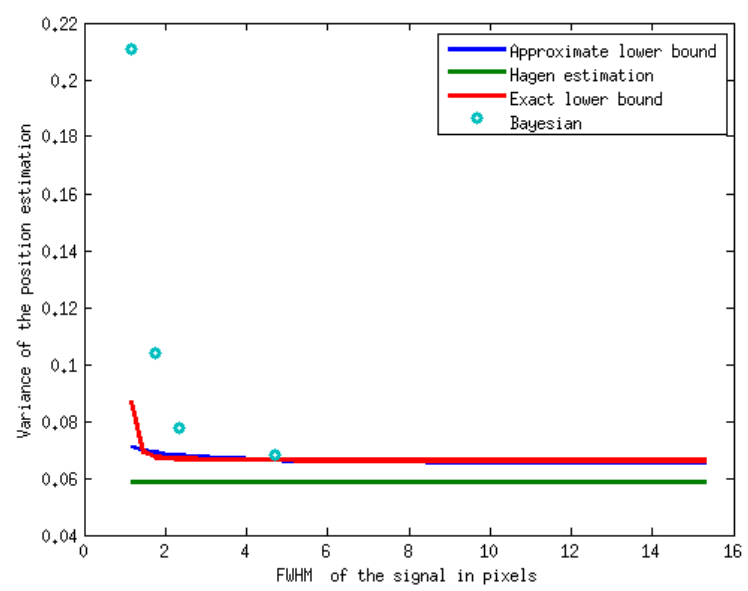

Figure 7: Convergence of the ML position estimation variance as a function of the FWHM for generated signal with $\mathrm{SNR}=5$

first signal is contained only in a few pixels with a high SNR, the second signal spreads over a large number of pixels with a high SNR. The third has the same shape as the first signal but the SNR here is much lower. The fourth one is as large as the second one but with a low SNR. According to Fig 9 representing the evaluation of the standard deviation of the signal position function of the 


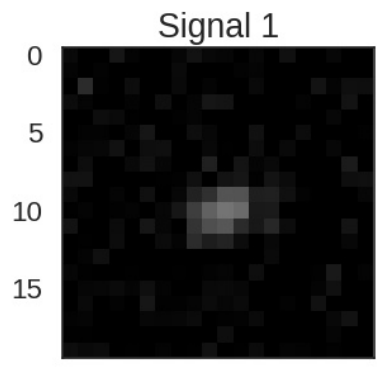

Signal 2

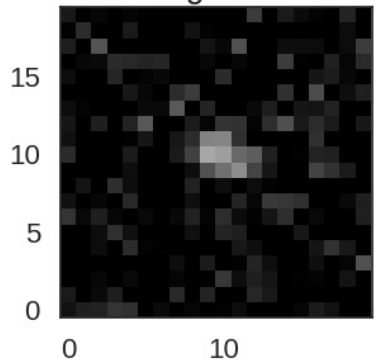

Signal 3

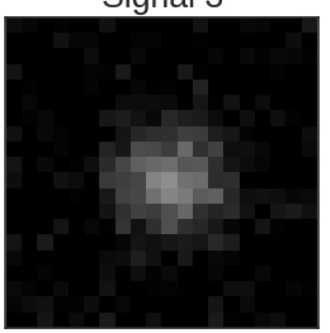

Signal 4

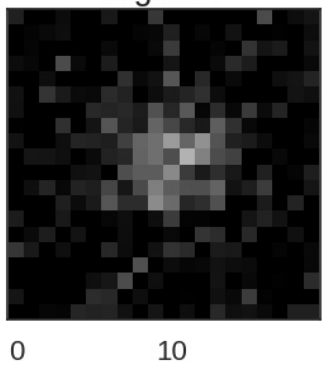

Figure 8: Example of Space object signals: signal 1 represents a small high SNR example, signal 2 represents a large high SNR example, signal 3 is a small low SNR example and signal 4 is a large low SNR example.

pixel resolution, large signals (signal 3 and 4) are less accurate than smaller ones (signal 1 and 2). In our case, the small low SNR (signal 2) still has a lower variance than signal 3 although there are more pixels to fit and less noise in signal 3.

\subsection{Comparison with Rule of Thumb}

A rule of thumb is often used in astronomy to roughly quantify the uncertainty in astrometric location. It defines the variance in astrometric position as:

$$
\widetilde{K_{x}}=\frac{1}{\left(F W H M_{x} \times S / N\right)^{2}}
$$

with an equivalent expression for the y axis. The rule of thumb captures the dependencies in the SNR well but fails to account for the influence of the signal geometry on the variance, as shown in Fig. 10. In this figure, the variances 


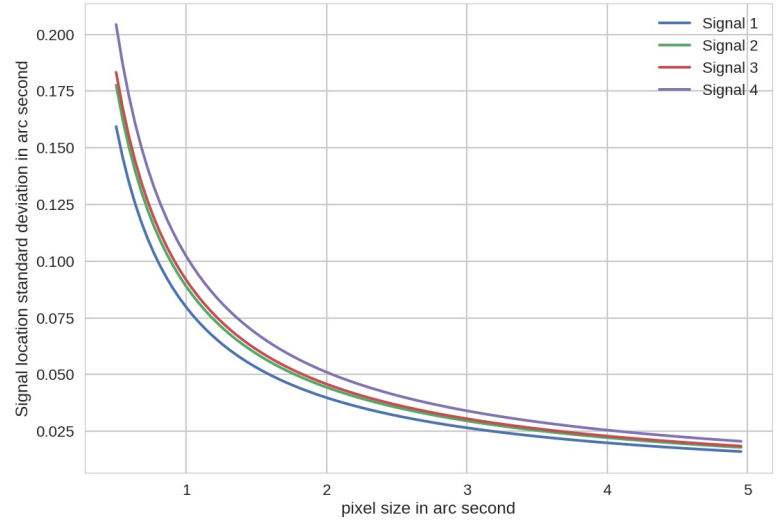

Figure 9: Evolution of the variance of the signal location with the resolution of the CCD for the signals shown on Fig 8

are computed with a constant signal to noise ratio of ten, and for different object image sizes of constant FWHM. The results clearly show that the rule of thumb underestimates the uncertainty in the astrometric position in any practical situations ie. as soon as the object image is larger than two or three pixels. The rule of thumb also wrongly goes to zero as the signal size increases, which contradicts the Monte Carlo simulations obtained in Fig. 6 . 


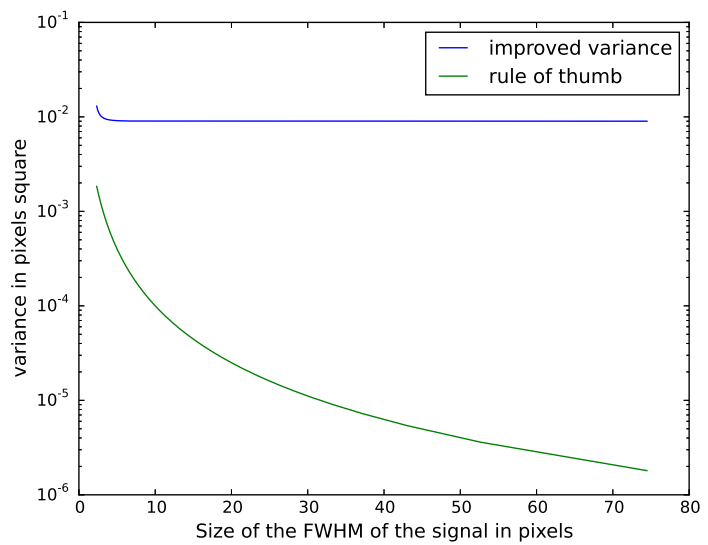

Figure 10: Comparison of the rule of thumb with our estimation of variance for different signal sizes.

\section{Conclusions}

As signal generation is a stochastic process, the identical incoming signal from an object leads to different object image representations. Each of the representations leads to a different centroid. The centroid is furthermore dependent on the the signal to noise ratio and the pixel size relative to the spread of the object image in pixels. To efficiently quantify the variation in the centroid estimates, this paper derives rigorous expressions to compute the variance of the extracted centroid, using the Rao Cramer lower bound (RCLB). While previous work has been shown to consistently underestimate the variance because of simplifying assumptions, in this work, improved analytic expressions for the variance have been derived. The obtain expressions depend on the signal to noise, the pixel size and the size of the object signal relative to the pixels have been derived. The new expressions consider that in realistic images, only a limited number of pixels are clearly above the background level, so-called cropping, instead of an unlimited amount of pixels. Furthermore, we do not assume that the object image center is centred within one pixel, but can have an arbitrary location within the brightest pixel as it is the case in actual images. The new expressions allow for fast 
computation of the variance. The derived expressions have been compared to a Bayesian evaluation using Markov Chain Monte Carlo (MCMC) method in the Metropolis Hasting implementation and a reference Monte Carlo estimation. The MCMC implementation is computationally intensive and performs similarly to the numerical evaluation of the RCLB and the analytical expression of the RCLB that has been derived in this paper albeit at much lower computational cost. Only when the full width of half maximum of the object signal reaches one pixel, i.e. when the signal that is above the background is mostly integrated in a single pixel, does the Bayesian MCMC approach shows advantages. Note that in most case the simple analytical expressions derived in this work compare very well to Bayesian and Monte Carlo calculations

In particular the domain of validity of the Gaussian noise assumption has been delimited. Using a Bayesian approach, it is shown that the distribution of the position estimation is well described by its first two moments. This result justifies the use of only the covariance matrix to quantify the uncertainties under consideration.

Using the derived analytical expressions, it has been shown that the observation likelihood can be computed analytically, solely based on the deterministic aspect of the observation process without expensive Monte Carlo simulations or other numerical procedures. Those results can directly be implemented in multitarget tracking algorithms to accurately evaluate the probability of detection knowing only the signal-to-noise ratio and the detection threshold.

For practical purposes, a simple shorthand lookup table has been created with precomputed variances.

\section{Acknowledgement}

The authors would like to thank Dr Emmanuel Delande for his advice on Bayesian estimation and maximum of likelihood estimation. We would like to acknowledge the support of this work via FA9550-14-1-0348 DEF. 
[1] S. B. Howell, Handbook of Ccd Astronomy, Cambridge University Press, 2006. doi:10.2277/0521617626.

[2] I. Smal, M. Loog, W. Niessen, E. Meijering, Quantitative comparison of spot detection methods in fluorescence microscopy, IEEE Transactions on Medical Imaging, 29 (2) (2010) 282-301.

[3] B. Zhang, J. Zerubia, J.-C. Olivo-Marin, Gaussian approximations of fluorescence microscope point-spread function models, Applied Optics 46 (10) (2007) 1819-1829.

[4] G. H. Massey, Philip; Jacoby, CCD Data: The Good, The Bad, and The Ugly, in: Steve B. Howell (Ed.), Astronomical CCD observing and reduction techniques, Vol. 23, Astronomical Society of the Pacific, San Francisco, 1992, p. 240.

[5] R. P. S. Mahler, Statistical Multisource-Multitarget Information Fusion, Artech House, Inc., Norwood, MA, USA, 2007.

[6] K. J. DeMars, I. I. Hussein, C. Frueh, M. K. Jah, R. Scott Erwin, MultipleObject Space Surveillance Tracking Using Finite-Set Statistics, Journal of Guidance, Control, and Dynamics (2015) 1-16.

[7] E. D. Delande, C. Frueh, J. Houssineau, D. E. Clark, Multi Object Filtering for Space Situational Awarness, AAS Space Flight Mechanics Meeting.

[8] J. S. McCabe, K. J. DeMars, C. Frueh, Integrated Detection and Tracking for Multiple Space Objects, AAS Space Flight Mechanics Meeting.

[9] W. J. Merline, S. B. Howell, A realistic model for point-sources imaged on array detectors: The model and initial results, Experimental Astronomy 6 (1-2) (1995) 163-210. doi:10.1007/BF00421131.

[10] B. E. A. Saleh, Estimation of the Location of an Optical Object with Photodetectors Limited by Quantum Noise, Appl. Opt. 13 (8) (1974) 18241827. doi:10.1364/A0.13.001824. 
[11] T. Delabie, J. D. Schutter, B. Vandenbussche, An accurate and efficient Gaussian fit centroiding algorithm for star trackers, Space Flight Mechanics Meeting, Kauai, Hawaii, 2013.

[12] A. Foi, M. Trimeche, V. Katkovnik, K. Egiazarian, Practical PoissonianGaussian noise modeling and fitting for single-image raw-data, IEEE transactions on image processing 17 (10).

[13] R. C. Stone, A comparison of digital centering algorithms, The Astronomical Journal 97 (4).

[14] R. Manish, Profile fitting in crowded astronomical images, Master's thesis, School of Aeronautics and Astronautics, Purdue University (2015).

[15] G. B. T. Schildknecht, U.Hugentobler A.Verdun, CCD Algorithms for space debris detection, Tech. rep., University of Berne (1995).

[16] H. Tiersch, S.B. Howell (ed.): Astronomical CCD observing and reduction techniques. Astronomical Society of the Pa-cific 1992, ASP Conference Series 23, 339 s., Preis: 55,-ISBN 0-937707-42-4, Astronomische Nachrichten 314 (6) (1993) 398. doi:10.1002/asna.2113140603.

[17] C. Frueh, Identification of Space Debris, Shaker Verlag, Aachen, 2011, iSBN: 978-3-8440-0516-5.

[18] J. R. Janesick, T. Elliott, S. Collins, M. M. Blouke, J. Freeman, Scientific charge-coupled devices, 1987. doi:10.1117/12.7974139.

[19] N. L, Hagen, D. Eustace, Gaussian profile estimation in two dimensions, Applied optics 47 (36) (2008) 6842-6851.

[20] N. Hagen, M. Kupinski, E. L. Dereniak, Gaussian profile estimation in one dimension, Applied optics 46 (22) (2007) 5374-5383.

[21] K. A. Winick, Cramer-Rao lower bounds on the performance of chargecoupled-device optical position estimators, Journal of Optical Society of America A 3 (11) (1986) 1809-1815. 
[22] F. Sanson, C. Frueh, Noise Quantification in Optical Observations of Resident Space Objects for Probability of Detection and Likelihood, in: Proc. AIAA/AAS Astrodynamic Specialist Conference, Vail, Colorado, 2015.

[23] F. Sanson, C. Frueh, Probability of detection in non-resolved images: Application to space object observation, Journal of Astronautical Sciences, submitted.

[24] C. Frueh, M. Jah, Detection Probability of Earth Orbiting Objects Using Optical Sensors, in: Advances in the Astronautical Sciences, Hilton Head, 2014.

[25] K. A. Winick, Cramer-Rao lower bounds on the performance of chargecoupled-device optical position estimators, JOSA A 3 (11) (1986) 1809-1815.

[26] A. Stuart, K. Ord, Kendall's Advanced Theory of Statistics: Volume 1: Distribution Theory, no. vol. $2^{\sim}$; vol. ${ }^{\sim} 1994$ in Kendall's Advanced Theory of Statistics, Wiley, 2009.

[27] H. H. Barrett, C. Dainty, D. Lara, Maximum-likelihood methods in wavefront sensing: stochastic models and likelihood functions, JOSA A 24 (2) (2007) 391-414.

[28] R. S. Savage, S. Oliver, Bayesian methods of astronomical source extraction, The Astrophysical Journal 661 (2) (2007) 1339.

[29] V. P. Bhapkar, Conditioning on ancillary statistics and loss of information in the presence of nuisance parameters, Journal of Statistical Planning and Inference 21 (2) (1989) 139-160.

[30] H. H. Barrett, K. J. Myers, Foundations of Image Science, Wiley Series in Pure and Applied Optics, Wiley, 2013.

[31] N. Hagen, E. Dereniak, Gaussian profile estimation in two dimensions., Applied optics 47 (36) (2008) 6842-6851. doi:10.1364/A0.47.006842. 
[32] J. Kaipio, E. Somersalo, Statistical and Computational Inverse Problems, Vol. 160 of Applied Mathematical Sciences, Springer-Verlag, New York, 2005. doi:10.1007/b138659.

[33] N. Metropolis, A. W. Rosenbluth, M. N. Rosenbluth, A. H. Teller, E. Teller, Equation of State Calculations by Fast Computing Machines, The Journal of Chemical Physics 21 (6) (1953) 1087. doi:10.1063/1.1699114.

[34] P. Diaconis, The markov chain monte carlo revolution, Bulletin of the American Mathematical Society 46 (2) (2009) 179-205.

\section{Nomenclature}

A Amplitude of the Gaussian

a size of the frame considered for Gaussian fitting in $\mathrm{x}$ direction

$B \quad$ Total intensity of the background pixels

$b \quad$ size of the frame considered for Gaussian fitting in y direction

$c_{1} \quad$ Gaussian parameter (cf Eq. 5 )

$c_{2} \quad$ Gaussian parameter (cf Eq. 5 )

$c_{3} \quad$ Gaussian parameter (cf Eq. 5 )

$c \quad$ Gaussian parameter in the symmetrical case $\left(c=c_{1}=c_{2}\right)$

$D \quad$ Determinant $\left(D=c_{1} c_{2}-c_{3}{ }^{2}\right)$

$D_{i} \quad$ Signal of the dark noise in pixel $i$

$E_{n, m} \quad E_{n, m}=e^{\left(-\frac{1}{2}\left(c_{1}\left(x_{n}-x_{0}\right)^{2}+2 c_{3}\left(x_{n}-x_{0}\right)\left(y_{m}-y_{0}\right)+c_{2}\left(y_{m}-y_{0}\right)^{2}\right)\right)}$

erf error function

F $\quad$ Fisher information

FWHM Full Width at Half Maximum

$g_{i} \quad$ Intensity at pixel $i$

$f_{i, j} \quad f_{i, j}(x, y)=x^{i} y^{j} \exp \left(-\left(c_{1} x^{2}+2 c_{3} x y+c_{2} y^{2}\right)\right)$

$\eta_{m} \quad \eta_{m}=y_{m}-y_{0}$

$\mathrm{G} \quad G=A e^{\left(-\frac{1}{2}\left(c_{1}\left(x_{i}-x_{0}\right)^{2}+2 c_{3}\left(x_{i}-x_{0}\right)\left(y_{i}-y_{0}\right)+c_{2}\left(y_{i}-y_{0}\right)^{2}\right)\right)}$

$\Gamma \quad$ Gamma function 


\begin{tabular}{|c|c|}
\hline$\delta_{x}$ & pixel size on $\mathrm{x}$ direction \\
\hline$\delta_{y}$ & pixel size on y direction \\
\hline$\delta$ & pixel size square pixels \\
\hline$\theta$ & vector of the Gaussian parameters $\left(A, c_{1}, c_{2}, c-3, x_{0}, y_{0}\right)$ \\
\hline$\theta_{\text {true }}$ & Actual value of the Gaussian parameters \\
\hline$\theta_{\mathrm{MLE}}$ & value of the Gaussian parameters estimated by the MLE \\
\hline$J_{x^{i} y^{j}}$ & $J_{x^{i} y^{j}}=\int_{-a+u_{x}}^{a+u_{x}} \int_{-b+u_{y}}^{b+u_{y}} x^{i} y^{j} \exp \left(-\left(c_{1} x^{2}+2 c_{3} x y+c_{2} y^{2}\right)\right) d x d y$ \\
\hline$K$ & Rao Cramer lower bound (inverse Fisher information) \\
\hline$\tilde{K}$ & centroid variance computed with the rule of thumb \\
\hline$L$ & Likelihood \\
\hline$l$ & log likelihood \\
\hline$\lambda_{\mathrm{D}, i}$ & Poisson parameter of the random variable $S_{D, i}$ \\
\hline$\lambda_{\text {obj }, i}$ & Poisson parameter of the random variable $S_{\mathrm{obj}, i}$ \\
\hline$\lambda_{S, i}$ & Poisson parameter of the random variable $S_{S, i}$ \\
\hline$\lambda_{\text {act }, i}$ & Poisson parameter of the random variable $S_{S, i}+S_{D, i}+S_{\mathrm{obj}, i}$ \\
\hline$\lambda_{b, d}$ & $\lambda_{b, d}=\lambda_{S}+\lambda_{D}$ \\
\hline$m$ & number of of sub-frames \\
\hline$\mu_{B}$ & Mean estimated background \\
\hline$N$ & Total Noise \\
\hline$N_{*}$ & Total noise due to the object \\
\hline$N_{x^{i} y^{j}}$ & $N_{x^{i} y^{j}}=\int_{\mathcal{R} \in} x^{i} y^{j} \exp \left(-\left(c_{1} x^{2}+2 c_{3} x y+c_{2} y^{2}\right)\right) d x d y$ \\
\hline$n_{B}$ & Number of pixel used in background determination \\
\hline$n_{\text {pix }}$ & Number of signal pixel \\
\hline$\xi_{n}$ & $\xi_{n}=x_{n}-x_{0}$ \\
\hline$S$ & Total Signal \\
\hline$S_{\mathrm{b}}$ & intensity of the brightest pixel \\
\hline$S_{*}$ & Averaged total signal of the object \\
\hline$d_{a}$ & $d_{a}=\exp \left(\frac{-a^{2} D}{c_{2}}\right)$ \\
\hline$d_{b}$ & $d_{b}=\exp \left(\frac{-b^{2} D}{c_{1}}\right)$ \\
\hline$S_{b}$ & Signal of the brightest pixel \\
\hline
\end{tabular}




$\begin{array}{ll}s & \text { Gaussian curve } \\ s_{i} & \text { Gaussian curve evaluated at pixel } i \\ \sigma & \text { CCD noise with the Gaussian fitting } \\ M_{x^{i} y^{j}} & \text { the surplus defined Eq. } 23 \\ t & \text { Detection threshold } \\ T & \text { integration interval } T=\left(-\infty ;-a+u_{x}\right] \cup\left[a+u_{x} ;+\infty\right) \\ Q & \times\left(-\infty ;-b+u_{y}\right] \cup\left[b+u_{y} ;+\infty\right) \\ U & Q=\sqrt{\pi} A^{2} \sqrt{D}\left(4 \frac{D^{2}}{\sqrt{c_{2} c_{1}}} a d_{a} b d_{b}-2 c_{1} c_{2}\left(\sqrt{\frac{D}{c_{2}}} a d_{a}+\sqrt{\frac{D}{c_{1}}} b d_{b}\right)+c_{1} \pi c_{2}\right) \delta_{x} \delta_{y} \\ \mathcal{U} & \text { Error associated to the CCD limited resolution } \\ u_{x} & \text { uniform distribution } \\ y_{m} & \text { Offset in } x \text { direction of the signal center with respect } \\ y_{0} & \text { to the pixel grid } \\ u_{y} & \text { Offset in } y \text { direction of the signal center with respect } \\ & \text { to the pixel grid } \\ & \text { center of the Gaussian on } \mathrm{x} \text { direction } \\ x_{3} & \end{array}$

\section{Appendix A. Lookup Table}

The following tables can be used to directly estimate the uncertainty in the signal astrometric position for a specific observation setting. $\frac{S_{b}}{N}$ is the signal to noise ratio of the brightest pixel, $n_{\text {pix }}$ corresponds to the number of pixel above half the maximum intensity, Ratio is the ratio of the longest direction over the shortest direction of the part of the signal with pixel intensity above half the brightest pixel intensity. Once those quantities have been determined the lookup table gives the variance in the astrometric position. The following table was obtain simulating Gaussian signal with fixed maximum intensity of 
1000 electrons and gain of 2. To obtain different values of signal-to-noise the background level varied between 10 and 1000 electrons and the readout noise varied between 0.2 to $150 \mathrm{ADU}$. 
Table A.1: LOOKUP TABLE for OBJECT ASTROMETRIC POSITION VARIANCE

\begin{tabular}{|l|l|l|l|l|l|}
\hline$\frac{S_{b}}{N}$ & $n_{\text {pix }}$ & Ratio & $\begin{array}{l}\text { Variance in } \\
\mathrm{x} \times \frac{\delta^{2}}{(S / N)^{2}}\end{array}$ & $\begin{array}{l}\text { Variance in } \\
\mathrm{y} \times \frac{\delta^{2}}{(S / N)^{2}}\end{array}$ & $\begin{array}{l}\text { Covariance } \\
\times \frac{\delta^{2}}{(S / N)^{2}}\end{array}$ \\
\hline 20.384705 & 69 & $\frac{11}{9}$ & 2.008388 & 2.008388 & -0.117197 \\
\hline 20.393602 & 24 & $\frac{9}{3}$ & 0.766788 & 5.317918 & -0.037245 \\
\hline 20.394506 & 25 & $\frac{10}{3}$ & 0.569103 & 7.263146 & -0.022204 \\
\hline 20.394879 & 23 & $\frac{11}{3}$ & 0.476102 & 8.800767 & -0.014129 \\
\hline 20.394970 & 10 & $\frac{9}{2}$ & 0.419734 & 10.118383 & -0.008623 \\
\hline 9.109910 & 74 & $\frac{11}{11}$ & 2.008388 & 2.008388 & -0.117197 \\
\hline 9.116150 & 22 & $\frac{10}{4}$ & 0.766788 & 5.317918 & -0.037245 \\
\hline 9.116785 & 23 & $\frac{11}{3}$ & 0.569103 & 7.263146 & -0.022204 \\
\hline 9.117046 & 17 & $\frac{9}{3}$ & 0.476102 & 8.800767 & -0.014129 \\
\hline 9.117110 & 15 & $\frac{10}{3}$ & 0.419734 & 10.118383 & -0.008623 \\
\hline 5.379920 & 52 & $\frac{10}{12}$ & 2.008388 & 2.008388 & -0.117197 \\
\hline 5.383823 & 24 & $\frac{12}{4}$ & 0.766788 & 5.317918 & -0.037245 \\
\hline 5.384219 & 25 & $\frac{12}{3}$ & 0.569103 & 7.263146 & -0.022204 \\
\hline 5.384383 & 17 & $\frac{12}{3}$ & 0.476102 & 8.800767 & -0.014129 \\
\hline 5.384423 & 10 & $\frac{9}{3}$ & 0.419734 & 10.118383 & -0.008623 \\
\hline 3.782134 & 44 & $\frac{12}{14}$ & 2.008388 & 2.008388 & -0.117197 \\
\hline 3.784920 & 22 & $\frac{13}{5}$ & 0.766788 & 5.317918 & -0.037245 \\
\hline 3.785203 & 14 & $\frac{9}{3}$ & 0.569103 & 7.263146 & -0.022204 \\
\hline 3.785319 & 13 & $\frac{11}{3}$ & 0.476102 & 8.800767 & -0.014129 \\
\hline 3.785348 & 20 & $\frac{13}{3}$ & 0.419734 & 10.118383 & -0.008623 \\
\hline 2.909595 & 32 & $\frac{13}{12}$ & 2.008388 & 2.008388 & -0.117197 \\
\hline 2.911750 & 22 & $\frac{12}{5}$ & 0.766788 & 5.317918 & -0.037245 \\
\hline 2.911969 & 10 & $\frac{10}{4}$ & 0.569103 & 7.263146 & -0.022204 \\
\hline 2.912060 & 9 & $\frac{10}{3}$ & 0.476102 & 8.800767 & -0.014129 \\
\hline 2.912082 & 11 & $\frac{10}{2}$ & 0.419734 & 10.118383 & -0.008623 \\
\hline
\end{tabular}




\section{Appendix B. Fisher Information}

The exact expression for the Fisher information as derived in [31] is:

$$
\begin{aligned}
& F_{11}=\sum_{n=0, m=0}^{n_{\text {pix }}} R_{n, m} \\
& F_{12}=A \sum_{n=0, m=0}^{n_{\text {pix }}} R_{n, m}\left(c_{1} \xi_{n}+c_{3} \eta_{m}\right) \\
& F_{13}=A \sum_{n=0, m=0}^{n_{\text {pix }}} R_{n, m}\left(c_{2} \eta_{m}+c_{3} \xi_{n}\right) \\
& F_{14}=-\frac{A}{2} \sum_{n=0, m=0}^{n_{\text {pix }}} R_{n, m} \xi_{n}^{2} \\
& F_{15}=-\frac{A}{2} \sum_{n=0, m=0}^{n_{\text {pix }}} R_{n, m} \eta_{m}^{2} \\
& F_{16}=-A \sum_{n=0, m=0}^{n_{\text {pix }}} R_{n, m} \xi_{n} \eta_{m} \\
& F_{22}=A^{2} \sum_{n=0, m=0}^{n_{\text {pix }}} R_{n, m}\left(c_{1} \xi_{n}+c_{3} \eta_{m}\right)^{2} \\
& F_{23}=A^{2} \sum_{n=0, m=0}^{n_{\text {pix }}} R_{n, m}\left(c_{2} \eta_{m}+c_{3} \xi_{n}\right)\left(c_{1} \xi_{n}+c_{3} \eta_{m}\right) \\
& F_{24}=-\frac{A^{2}}{2} \sum_{n=0, m=0}^{n_{\text {pix }}} R_{n, m} \xi_{n}^{2}\left(c_{1} \xi_{n}+c_{3} \eta_{m}\right) \\
& F_{25}=-\frac{A^{2}}{2} \sum_{n=0, m=0}^{n_{\text {pix }}} R_{n, m} \eta_{m}^{2}\left(c_{1} \xi_{n}+c_{3} \eta_{m}\right) \\
& F_{26}=-A^{2} \sum_{n=0, m=0}^{n_{\text {pix }}} R_{n, m} \xi_{n} \eta_{m}\left(c_{1} \xi_{n}+c_{3} \eta_{m}\right) \\
& F_{33}=A^{2} \sum_{n=0, m=0}^{n_{\text {pix }}} R_{n, m}\left(c_{2} \eta_{m}+c_{3} \xi_{n}\right)^{2} \\
& F_{34}=-\frac{A^{2}}{2} \sum_{n=0, m=0}^{n_{\text {pix }}} R_{n, m} \xi_{n}^{2}\left(c_{2} \eta_{m}+c_{3} \xi_{n}\right)
\end{aligned}
$$




$$
\begin{aligned}
& F_{35}=-\frac{A^{2}}{2} \sum_{n=0, m=0}^{n_{\text {pix }}} R_{n, m} \eta_{m}^{2}\left(c_{2} \eta_{m}+c_{3} \xi_{n}\right) \\
& F_{36}=-A^{2} \sum_{n=0, m=0}^{n_{\text {pix }}} R_{n, m} \xi_{n} \eta_{m}\left(c_{2} \eta_{m}+c_{3} \xi_{n}\right) \\
& F_{44}=\frac{A^{2}}{4} \sum_{n=0, m=0}^{n_{\text {pix }}} R_{n, m} \xi_{n}^{4} \\
& F_{45}=\frac{A^{2}}{4} \sum_{n=0, m=0}^{n_{\text {pix }}} R_{n, m} \xi_{n}^{2} \eta_{m}^{2} \\
& F_{46}=\frac{A^{2}}{2} \sum_{n=0, m=0}^{n_{\text {pix }}} R_{n, m} \xi_{n}^{3} \eta_{m} \\
& F_{55}=\frac{A^{2}}{4} \sum_{n=0, m=0}^{n_{\text {pix }}} R_{n, m} \eta_{m}^{4} \\
& F_{56}=\frac{A^{2}}{2} \sum_{n=0, m=0}^{n_{\text {pix }}} R_{n, m} \eta_{m}^{3} \xi_{n} \\
& F_{66}=A^{2} \sum_{n=0, m=0}^{n_{\text {pix }}} R_{n, m} \xi_{n}^{2} \eta_{m}^{2},
\end{aligned}
$$

where $R_{n, m}=\frac{\delta_{x}^{2} \delta_{y}^{2} E_{n, m}^{2}}{2 \sigma^{2}}$ and

$E_{n, m}=\exp \left(-\frac{1}{2}\left(c_{1}\left(x_{n}-x_{0}\right)^{2}+2 c_{3}\left(x_{n}-x_{0}\right)\left(y_{m}-y_{0}\right)+c_{2}\left(y_{m}-y_{0}\right)^{2}\right)\right) \cdot \xi_{n}=$ $x_{n}-x_{0}$ and $\eta_{m}=y_{m}-y_{0}$.

\section{Appendix C. Derivation of the odd integrals}

We start from :

$$
J_{x^{i} y^{j}}=\int_{a-u_{x}}^{a+u_{x}} \int_{-b}^{b} f_{i, j}(x, y) d x d y+\int_{-a}^{a} \int_{b-u_{y}}^{b+u_{y}} f_{i, j}(x, y) d x d y
$$

With $f_{i, j}(x, y)=x^{i} y^{j} \exp \left(-\frac{1}{2}\left(c_{1} x^{2}+2 c_{3} x y+c_{2} y^{2}\right)\right)$. The expression of $J_{x^{i} y^{j}}$ is computed by integrating by part to get rid of the polynomial factor and then integrating the univariate Gaussian function using the error function. Finally 
we get :

$$
\begin{aligned}
J_{x}= & -\frac{u_{y} b c_{3} \pi}{c 1^{3 / 2}} \exp \left(-\frac{D b^{2}}{c_{1}}\right)\left(\operatorname{erf}\left(\frac{a c_{1}+b c_{3}}{\sqrt{c_{1}}}\right)+\operatorname{erf}\left(\frac{b c_{3}}{\sqrt{c_{1}}}\right)\right) \\
& +\frac{u_{y}}{c_{1}} \exp \left(-c_{1} a^{2}-c_{2} b^{2}-2 c_{3} a b\right) \\
J_{x^{3}}= & -2 \frac{u_{y} b c_{3} \pi^{1 / 2}}{c 1^{5 / 2}}\left(\frac{3}{4}+\frac{c_{3}^{2} b^{2}}{2 c_{1}}\right) \\
& \times \exp \left(-\frac{D b^{2}}{c_{1}}\right)\left(\operatorname{erf}\left(\frac{a c_{1}+b c_{3}}{\sqrt{c_{1}}}\right)+e r f\left(\frac{a c_{1}-b c_{3}}{\sqrt{c_{1}}}\right)\right) \\
& +\frac{u_{x} a^{3} \sqrt{\pi}}{c_{2}^{1 / 2}} \exp \left(-\frac{D a^{2}}{c_{2}}\right)\left(\operatorname{erf}\left(\frac{b c_{2}+a c_{3}}{\sqrt{c_{2}}}\right)+\operatorname{erf}\left(\frac{b c_{2}-a c_{3}}{\sqrt{c_{2}}}\right)\right) \\
J_{x y^{2}}= & \frac{u_{y} b^{3} c_{3} \pi^{1 / 2}}{c 1^{3 / 2}} \exp \left(-\frac{D b^{2}}{c_{1}}\right)\left(\operatorname{erf}\left(\frac{a c_{1}+b c_{3}}{\sqrt{c_{1}}}\right)+\operatorname{erf}\left(\frac{a c_{1}-b c_{3}}{\sqrt{c_{1}}}\right)\right) \\
+ & \frac{u_{x} a^{3} c_{3}^{2} \pi^{1 / 2}}{c 2^{5 / 2}} \exp \left(-\frac{D a^{2}}{c_{2}}\right)\left(\operatorname{erf}\left(\frac{b c_{2}+a c_{3}}{\sqrt{c_{2}}}\right)+\operatorname{erf}\left(\frac{b c_{2}-a c_{3}}{\sqrt{c_{2}}}\right)\right) \\
+ & \frac{u_{x} a \pi^{1 / 2}}{2 c 2^{3 / 2}} \exp \left(-\frac{D a^{2}}{c_{2}}\right)\left(\operatorname{erf}\left(\frac{b c_{2}+a c_{3}}{\sqrt{c_{2}}}\right)+\operatorname{erf}\left(\frac{b c_{2}-a c_{3}}{\sqrt{c_{2}}}\right)\right)
\end{aligned}
$$

Equivalent results are obtained for $J_{y}, J_{y^{3}}, J_{x^{2} y}$.

\section{Appendix D. Illustration computation of e $J_{x^{i} y^{j}}$ (Eq. 14) for even case $(i+j$ even $)$}

The general strategy is to use firstly to compute $\tilde{M}_{x^{i} y^{j}}$ defined in eq. 25 secondly to use $N_{x^{i} y^{j}}$ given in Appendix E and then to compute $J_{x^{i} y^{j}}$ using 20 and the fact that $\tilde{M}_{x^{i} y^{j}} \simeq M_{x^{i} y^{j}}$. Since $N_{x^{i} y^{j}}$ is already given in [19] most of the work is on computing $\tilde{M}_{x^{i} y^{j}}$. 
case $\mathbf{i}=\mathbf{2}, \mathbf{j}=\mathbf{0}$ We first compute $\tilde{M}_{x^{2}}$. According to Eq. 24 we have :

$$
\begin{aligned}
\tilde{M}_{x^{2}}= & 2 \int_{(-\infty ;-a] \times R} x^{2} \exp \left(-\left(c_{1} x^{2}+2 c_{3} x y+c_{2} y^{2}\right)\right) d x d y \\
& +2 \int_{R \times(-\infty ;-b]} x^{2} \exp \left(-\left(c_{1} x^{2}+2 c_{3} x y+c_{2} y^{2}\right)\right) d x d y .
\end{aligned}
$$

We integrate along one axis in each integral and get :

$$
\begin{aligned}
\tilde{M}_{x^{2}}= & 2 \sqrt{\frac{\pi}{c_{2}}} \int_{-\infty}^{-a} x^{2} \exp \left(-\left(x^{2} \frac{D}{c_{2}}\right)\right) d x \\
+ & 2 \frac{c_{3}^{2} \sqrt{\pi}}{c_{1}^{5 / 2}} \int_{-\infty}^{-b} y^{2} \exp \left(-\left(\frac{y^{2} D}{c_{1}}\right)\right) d y \\
& +\frac{\sqrt{\pi}}{c_{1}^{3 / 2}} \int_{-\infty}^{-b} \exp \left(-\left(\frac{y^{2} D}{c_{1}}\right)\right) d y .
\end{aligned}
$$

Each term can be simplified using integration by part:

$$
\begin{aligned}
2 \sqrt{\frac{\pi}{c_{2}}} \int_{-\infty}^{-a} x^{2} e^{\left(-\left(x^{2} \frac{D}{c_{2}}\right)\right)} d x & =-\frac{\sqrt{\pi c_{2}}}{D}\left[x e^{\left(-\frac{x^{2} D}{c_{2}}\right)}\right]_{-\infty}^{-a}+\frac{c_{2}}{D} \sqrt{\frac{\pi}{c_{2}}} \int_{-\infty}^{-a} e^{\left(-\frac{x^{2} D}{c_{2}}\right)} d x \\
& =\frac{a \sqrt{\pi c_{2}}}{D} e^{\left(-\frac{a^{2} D}{c_{2}}\right)}+\frac{c_{2}}{D} \sqrt{\frac{\pi}{c_{2}}} \int_{-\infty}^{-a} e^{\left(-\frac{x^{2} D}{c_{2}}\right)} d x
\end{aligned}
$$

and

$$
\begin{gathered}
2 \frac{c_{3}^{2} \sqrt{\pi}}{c_{1}^{5 / 2}} \int_{-\infty}^{-b} y^{2} \exp \left(-\left(\frac{y^{2} D}{c_{1}}\right)\right) d y \\
=\frac{c_{3}^{2} b \sqrt{\pi}}{D c_{1}^{3 / 2}} \exp \left(\frac{-b^{2} D}{c_{1}}\right)+\frac{\sqrt{\pi} c_{3}^{2}}{D c_{1}^{3 / 2}} \int_{-\infty}^{-b} \exp \left(-\frac{y^{2} D}{c_{1}}\right) d y .
\end{gathered}
$$

So finally summing everything leads to:

$$
\begin{gathered}
\tilde{M}_{x^{2}}=\frac{a \sqrt{\pi c_{2}}}{D} e^{\left(-\frac{a^{2} D}{c_{2}}\right)}+\frac{c_{2}}{D} \sqrt{\frac{\pi}{c_{2}}} \int_{-\infty}^{-a} e^{\left(-\frac{x^{2} D}{c_{2}}\right)} d x \\
+\frac{c_{3}^{2} b \sqrt{\pi}}{D c_{1}^{3 / 2}} e^{\left(\frac{-b^{2} D}{c_{1}}\right)}+\left(\frac{\sqrt{\pi} c_{3}^{2}}{D c_{1}^{3 / 2}}+\frac{\sqrt{\pi}}{c_{2}^{3 / 2}}\right) \int_{-\infty}^{-b} e^{\left(-\frac{y^{2} D}{c_{1}}\right)} d y .
\end{gathered}
$$

Using a integration by part and under the assumption that $a$ and $b$ are large relative to $\sqrt{\frac{c_{2}}{D}}$ and $\sqrt{\frac{c_{1}}{D}}$ leads to:

$$
\int_{-\infty}^{-a} \exp \left(-x^{2} \frac{D}{c 2}\right) \simeq \frac{c_{2}}{2 D a} \exp \left(\frac{-a^{2} D}{c_{2}}\right)
$$


This can be used to simplify D.5.

$$
\begin{aligned}
\tilde{M}_{x^{2}}= & \frac{a \sqrt{\pi c_{2}}}{D} \exp \left(-\frac{a^{2} D}{c_{2}}\right)+\frac{c_{2}^{3 / 2}}{2 a D^{2}} \sqrt{\pi} \exp \left(-\frac{a^{2} D}{c_{2}}\right) \\
& +\frac{c_{3}^{2} b \sqrt{\pi}}{D c_{1}^{3 / 2}} \exp \left(\frac{-b^{2} D}{c_{1}}\right) \\
& +\left(\frac{\sqrt{\pi} c_{3}^{2}}{D c_{1}^{3 / 2}}+\frac{\sqrt{\pi}}{c_{2}^{3 / 2}}\right) \frac{c_{1}}{2 D b} \exp \left(-\frac{b^{2} D}{c_{1}}\right) .
\end{aligned}
$$

Reordering the terms:

$$
\begin{aligned}
\tilde{M}_{x^{2}} & =\exp \left(-\frac{a^{2} D}{c_{2}}\right)\left(\frac{a \sqrt{\pi c_{2}}}{D}+\frac{c_{2}^{3 / 2}}{2 a D^{2}} \sqrt{\pi}\right) \\
& +\exp \left(-\frac{b^{2} D}{c_{1}}\right)\left(\frac{c_{3}^{2} b \sqrt{\pi}}{D c_{1}^{3 / 2}}+\left(\frac{\sqrt{\pi} c_{3}^{2}}{D c_{1}^{3 / 2}}+\frac{\sqrt{\pi}}{c_{2}^{3 / 2}}\right) \frac{c_{1}}{2 D b}\right) .
\end{aligned}
$$

Finally the truncated signal is ( assuming $\tilde{M}_{x^{2}}=M_{x^{2}}$ )

$$
J_{x^{2}}=N_{x^{2}}-\tilde{M}_{x^{2}},
$$

so we get :

$$
\begin{aligned}
J_{x^{2}} & =\frac{\pi c_{2}}{2 D^{3 / 2}}-\exp \left(-\frac{a^{2} D}{c_{2}}\right)\left(\frac{a \sqrt{\pi c_{2}}}{D}+\frac{c_{2}^{3 / 2}}{2 a D^{2}} \sqrt{\pi}\right) \\
& -\exp \left(-\frac{b^{2} D}{c_{1}}\right)\left(\frac{c_{3}^{2} b \sqrt{\pi}}{D c_{1}^{3 / 2}}+\left(\frac{\sqrt{\pi} c_{3}^{2}}{D c_{1}^{3 / 2}}+\frac{\sqrt{\pi}}{c_{2}^{3 / 2}}\right) \frac{c_{1}}{2 D b}\right) .
\end{aligned}
$$

By exchanging the role of $x$ and $y$ in the previous derivation, an expression for $J_{y^{2}}$ is:

$$
\begin{aligned}
J_{y^{2}} & =\frac{\pi c_{1}}{2 D^{3 / 2}}-\exp \left(-\frac{b^{2} D}{c_{1}}\right)\left(\frac{b \sqrt{\pi c_{1}}}{D}+\frac{c_{1}^{3 / 2}}{2 b D^{2}} \sqrt{\pi}\right) \\
& -\exp \left(-\frac{a^{2} D}{c_{2}}\right)\left(\frac{c_{3}^{2} a \sqrt{\pi}}{D c_{2}^{3 / 2}}+\left(\frac{\sqrt{\pi} c_{3}^{2}}{D c_{2}^{3 / 2}}+\frac{\sqrt{\pi}}{c_{1}^{3 / 2}}\right) \frac{c_{2}}{2 D a}\right) .
\end{aligned}
$$

case $\mathrm{i}=0, \mathrm{j}=\mathbf{0}$

The calculations are very similar for $\tilde{M} \cdot \tilde{M}$ is defined as:

$$
\begin{aligned}
\tilde{M}= & 2 \int_{(-\infty ;-a] \times R} \exp \left(-\left(c_{1} x^{2}+2 c_{3} x y+c_{2} y^{2}\right)\right) d x d y \\
& +2 \int_{R \times(-\infty ;-b]} \exp \left(-\left(c_{1} x^{2}+2 c_{3} x y+c_{2} y^{2}\right)\right) d x d y
\end{aligned}
$$


And when integrating over $y$ for for the first integral and $x$ for the second one gets:

$$
\begin{aligned}
\tilde{M}= & 2 \sqrt{\frac{\pi}{c_{2}}} \int_{[-\infty ;-a]} \exp \left(-\left(\frac{D}{c_{2}} x^{2}\right)\right) d x d y \\
& +2 \sqrt{\frac{\pi}{c_{1}}} \int_{(-\infty ;-b]} \exp \left(-\left(\frac{D}{c_{1}} y^{2}\right)\right) d x d y
\end{aligned}
$$

Using the approximation in (D.6) we get:

$$
\tilde{M}=\frac{\sqrt{\pi c_{2}}}{D a} \exp \left(-\frac{D}{c_{2}} a^{2}\right)+\frac{\sqrt{\pi c_{1}}}{D b} \exp \left(-\frac{D}{c_{1}} b^{2}\right)
$$

Case $\mathrm{i}=\mathbf{1} \mathrm{j}=1$

The same technique is used to compute the surplus $\tilde{M}_{x, y}$ defined in Eq. 21 . In the first step:

$$
\begin{array}{r}
\int_{-\infty}^{-a} \int_{-\infty}^{\infty} x y \exp \left(-c_{1} x^{2}-c_{2} y^{2}-2 c_{3} x y\right) d x d y \\
=-\frac{\sqrt{\pi} c_{3}}{2 D \sqrt{c_{2}}} \exp \left(-\frac{D a^{2}}{c 2}\right)\left(a+\frac{c_{2}}{2 \sqrt{D} a}\right) .
\end{array}
$$

By exchanging the role of $x$ and $y$ in the previous equation we get:

$$
\begin{aligned}
\int_{-\infty}^{-\infty} & \int_{-\infty}^{-b} x y \exp \left(-c_{1} x^{2}-c_{2} y^{2}-2 c_{3} x y\right) d x d y \\
& =-\frac{\sqrt{\pi} c_{3}}{2 D \sqrt{c_{1}}} \exp \left(-\frac{D b^{2}}{c 1}\right)\left(b+\frac{c_{1}}{2 \sqrt{D} b}\right) .
\end{aligned}
$$

So the surplus is approximately:

$$
\begin{aligned}
\tilde{M}_{x y} & =-\frac{\sqrt{\pi} c_{3}}{D \sqrt{c_{1}}} \exp \left(-\frac{D b^{2}}{c 1}\right)\left(b+\frac{c_{1}}{2 \sqrt{D} b}\right) \\
& -\frac{\sqrt{\pi} c_{3}}{D \sqrt{c_{2}}} \exp \left(-\frac{D a^{2}}{c 2}\right)\left(a+\frac{c_{2}}{2 \sqrt{D} a}\right)
\end{aligned}
$$

Using Eq. 20, we obtain the following expression :

$$
\begin{aligned}
J_{x y} & =N_{x y}+\frac{\sqrt{\pi} c_{3}}{D \sqrt{c_{1}}} \exp \left(-\frac{D b^{2}}{c 1}\right)\left(b+\frac{c_{1}}{2 \sqrt{D} b}\right) \\
& +\frac{\sqrt{\pi} c_{3}}{D \sqrt{c_{2}}} \exp \left(-\frac{D a^{2}}{c 2}\right)\left(a+\frac{c_{2}}{2 \sqrt{D} a}\right) \\
& =-\frac{c_{3} \pi}{2 D^{3 / 2}}+\frac{\sqrt{\pi} c_{3}}{D \sqrt{c_{1}}} \exp \left(-\frac{D b^{2}}{c 1}\right)\left(b+\frac{c_{1}}{2 \sqrt{D} b}\right) \\
& +\frac{\sqrt{\pi} c_{3}}{D \sqrt{c_{2}}} \exp \left(-\frac{D a^{2}}{c 2}\right)\left(a+\frac{c_{2}}{2 \sqrt{D} a}\right) .
\end{aligned}
$$


where $N_{x y}$ is given in Appendix $\mathrm{E}$

Unfortunately, those expressions remain complex so it can be advantageous to keep the dominant terms and get :

$$
\begin{aligned}
& J_{x^{2}}=N_{x^{2}}-\frac{a \sqrt{\pi c_{2}}}{D} \exp \left(-\frac{a^{2} D}{c_{2}}\right)-\frac{c_{3}^{2} b \sqrt{\pi}}{D c_{1}^{3 / 2}} \exp \left(-\frac{b^{2} D}{c_{1}}\right) \\
& J_{y^{2}}=N_{y^{2}}-\frac{b \sqrt{\pi c_{1}}}{D} \exp \left(-\frac{b^{2} D}{c_{1}}\right)-\frac{c_{3}^{2} a \sqrt{\pi}}{D c_{2}^{3 / 2}} \exp \left(-\frac{a^{2} D}{c_{2}}\right) \\
& J_{x y}=N_{x y}+\frac{\sqrt{\pi} b c_{3}}{D \sqrt{c_{1}}} \exp \left(-\frac{D b^{2}}{c 1}\right)+\frac{\sqrt{\pi} a c_{3}}{D \sqrt{c_{2}}} \exp \left(-\frac{D a^{2}}{c 2}\right)
\end{aligned}
$$

The computation of $J_{x^{2}}, J_{y^{2}}, J_{x y}$ is sufficient to compute $F_{2,2}, F_{2,3}$ and $F_{3,3}$.

Case $\mathrm{i}=\mathbf{2} \mathrm{j}=\mathbf{2}$

We follow the same procedure as in the previous paragraph by computing. We have: $\tilde{M}_{x^{2} y^{2}}$ :

$$
\begin{aligned}
\int_{-\infty}^{-a} \int_{-\infty}^{\infty} x^{2} y^{2} \exp \left(-c_{1} x^{2}-c_{2} y^{2}-2 c_{3} x y\right) d x d y \\
=\frac{2 \pi c_{3}^{2}+\pi c_{2} c_{1}}{8 D^{3 / 2}}\left(1-\operatorname{erf}\left(\frac{a \sqrt{D}}{\sqrt{c_{2}}}\right)\right) \\
+\frac{\sqrt{\pi} c_{3}^{2} a^{3}}{2 D c_{2}^{3 / 2}} \exp \left(-\frac{a^{2} D}{c_{2}}\right)+\frac{2 a D^{1 / 2}}{\sqrt{\pi c_{2}}} \pi \frac{2 c_{3}+c_{1} c_{2}}{4 D^{5 / 2}}
\end{aligned}
$$

and also:

$$
\begin{aligned}
& \int_{-\infty}^{-a} \int_{-\infty}^{\infty} x^{2} y^{2} \exp \left(-c_{1} x^{2}-c_{2} y^{2}-2 c_{3} x y\right) d x d y \\
& \quad=\frac{2 \pi c_{3}^{2}+\pi c_{2} c_{1}}{8 D^{3 / 2}}\left(1-\operatorname{erf}\left(\frac{b \sqrt{D}}{\sqrt{c_{1}}}\right)\right)+\frac{\sqrt{\pi} c_{3}^{2} b^{3}}{2 D c_{1}^{3 / 2}} \exp \left(-\frac{b^{2} D}{c_{1}}\right) \\
& \quad+\frac{2 b D^{1 / 2}}{\sqrt{\pi c_{1}}} \pi \frac{2 c_{3}+c_{1} c_{2}}{4 D^{5 / 2}} \exp \left(-\frac{b^{2} D}{c_{1}}\right) .
\end{aligned}
$$


So the surplus becomes:

$$
\begin{aligned}
\tilde{M}_{x^{2} y^{2}}= & \frac{2 \pi c_{3}^{2}+\pi c_{2} c_{1}}{4 D^{3 / 2}}\left(2-\operatorname{erf}\left(\frac{a \sqrt{D}}{\sqrt{c_{2}}}\right)-\operatorname{erf}\left(\frac{b \sqrt{D}}{\sqrt{c_{1}}}\right)\right) \\
& +\frac{\sqrt{\pi} c_{3}^{2} b^{3}}{D c_{1}^{3 / 2}} \exp \left(-\frac{b^{2} D}{c_{1}}\right)+\frac{4 b D^{1 / 2}}{\sqrt{\pi c_{1}}} \pi \frac{2 c_{3}+c_{1} c_{2}}{4 D^{5 / 2}} \exp \left(-\frac{b^{2} D}{c_{1}}\right) \\
& +\frac{\sqrt{\pi} c_{3}^{2} a^{3}}{D c_{2}^{3 / 2}} \exp \left(-\frac{a^{2} D}{c_{2}}\right)+\frac{4 a D^{1 / 2}}{\sqrt{\pi c_{2}}} \pi \frac{2 c_{3}+c_{1} c_{2}}{4 D^{5 / 2}}
\end{aligned}
$$

where we used the approximation:

$$
1-\operatorname{erf}\left(a \frac{\sqrt{D}}{\sqrt{c_{2}}}\right) \simeq \frac{\sqrt{c_{2}}}{\sqrt{\pi D} a} \exp \left(-a^{2} \frac{D}{c_{2}}\right) .
$$

To obtain a compact form for $\tilde{M}_{x^{2}, y^{2}}$ we can plug in $N_{x^{2} y^{2}}$ from Appendix E in Eq. D.23 and get:

$$
\begin{aligned}
\tilde{M}_{x^{2} y^{2}}= & \frac{D N_{x^{2} y^{2}}}{3 \sqrt{\pi}}\left(\frac{\sqrt{c_{2}}}{a \sqrt{D}} \exp \left(-\frac{a^{2} D}{c_{2}}\right)+\frac{\sqrt{c_{1}}}{b \sqrt{D}} \exp \left(-\frac{b^{2} D}{c_{1}}\right)\right) \\
& +\frac{4}{3 \sqrt{\pi}} N_{x^{2} y^{2}}\left(\frac{a D^{1 / 2}}{\sqrt{c_{2}}} \exp \left(-\frac{a^{2} D}{c_{2}}\right)+\frac{b D^{1 / 2}}{\sqrt{c_{1}}} \exp \left(-\frac{b^{2} D}{c_{1}}\right)\right) \\
& +\frac{\sqrt{\pi} c_{3}^{2} b^{3}}{D c_{2}^{3 / 2}} \exp \left(-\frac{b^{2} D}{c_{1}}\right)+\frac{\sqrt{\pi} c_{3}^{2} a^{3}}{D} \exp \left(-\frac{a^{2} D}{c_{2}}\right)
\end{aligned}
$$

\section{case $i=4 j=0$}

We follow the same procedure as in the previous paragraph by computing $\tilde{M}_{x^{4}}$.

We have :

$$
\begin{array}{r}
\int_{-\infty}^{-a} \int_{-\infty}^{-\infty} x^{4} \exp \left(-c_{1} x^{2}-c_{2} y^{2}-2 c_{3} x y\right) d x d y=\frac{3 c_{2}^{2} \sqrt{\pi c_{2}}}{8 D^{3} a} \exp \left(-a^{2} \frac{D}{c_{2}}\right) \\
-\frac{\sqrt{\pi} a^{3} c_{3}^{2} \sqrt{c_{2}}}{2 D^{2}} \exp \left(-\frac{a^{2} D}{c_{2}}\right)+\frac{3 c_{2}^{3 / 2} a \sqrt{\pi}}{4 D^{2}} \exp \left(-\frac{a^{2} D}{c_{2}}\right)+\frac{a^{3} c_{1} c_{2}^{3 / 2} \sqrt{\pi}}{2 D^{2}} \exp \left(-\frac{a^{2} D}{c_{2}}\right)
\end{array}
$$

and

$$
\begin{gathered}
\int_{-\infty}^{\infty} \int_{-\infty}^{-b} x^{4} \exp \left(-c_{1} x^{2}-c_{2} y^{2}-2 c_{3} x y\right) d x d y=\frac{c_{3} b^{3} \pi^{1 / 2}}{c_{1}^{1 / 2} D^{1 / 2}} \exp \left(-\frac{D b^{2}}{c_{1}}\right) \\
-\frac{3 c_{3} b \sqrt{c_{1} \pi}}{4 D^{2}} \exp \left(-\frac{D b^{2}}{c_{1}}\right)+\frac{3}{4} \frac{\sqrt{\pi} c_{1}^{3 / 2} c_{3}}{b D^{3}} \exp \left(-\frac{D b^{2}}{c_{1}}\right)
\end{gathered}
$$


So the surplus becomes:

$$
\begin{aligned}
\tilde{M}_{x^{4}}= & 2\left(\frac{1}{2} \frac{\sqrt{\pi} b^{3} c_{3}^{4}}{c_{1}^{7 / 2} D}+\frac{3 c_{3}^{2} c_{2} b \sqrt{\pi}}{2 c_{1}^{3 / 2} D^{2}}-\frac{3 b \pi^{1 / 2} c_{3}^{4}}{4 c_{1}^{5 / 2} D^{2}}-\frac{3 \sqrt{\pi c_{1}} c_{2}^{2}}{4 D^{3} b}\right) \exp \left(-\frac{b^{2} D}{c_{1}}\right) \\
& +2\left(\frac{3 c_{2}^{2} \sqrt{\pi c_{2}}}{8 D^{3} a}+\frac{3 c_{2}^{3 / 2} a \sqrt{\pi}}{4 D^{2}}+\frac{a^{3} c_{2}^{1 / 2} \sqrt{\pi}}{2 D}\right) \exp \left(-\frac{a^{2} D}{c_{2}}\right)
\end{aligned}
$$

$J_{x^{4}}$ is then computed using equation 20 and the expression for $N_{x^{4}}$ given in Appendix E.

\section{Appendix E. Expressions for the $N_{x, y}$}

The expressions for $N_{x^{i}, y^{j}}$ are classical and already derived in [19]. They are recalled here for completeness

$$
\begin{aligned}
& N_{x}=0 . \\
& N_{x^{3}}=0 \\
& N_{y^{3}}=0 \\
& N_{x y^{2}}=0 \\
& N_{x^{2} y}=0 \\
& N_{x^{3} y}=0 \\
& N_{y^{3} x}=0 \\
& N=\frac{\pi}{\sqrt{D}}
\end{aligned}
$$




$$
\begin{aligned}
N_{x^{2}} & =\frac{1}{2} \frac{\pi c_{2}}{D^{3 / 2}}, \\
N_{y^{2}} & =\frac{1}{2} \frac{\pi c_{1}}{D^{3 / 2}}, \\
N_{y^{4}} & =\frac{3}{4} \frac{c_{1}^{2} \pi}{D^{5 / 2}}, \\
N_{x^{4}} & =\frac{3}{4} \frac{c_{2}^{2} \pi}{D^{5 / 2}}, \\
N_{x y} & =-\frac{1}{2} \frac{c_{3} \pi}{D^{3 / 2}}, \\
N_{x^{2} y^{2}}=\frac{3}{4} & \frac{\pi}{c_{1}}
\end{aligned}
$$

